\title{
Dieter Strauch \\ Die Entwicklung des Rheinschiffahrtsrechts zwischen 1815 und 1868
}

\section{Inhalt}

I. KURZER BLICK IN DIE VORGESCHICHTE .....................................................2

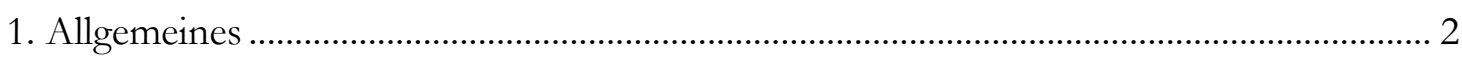

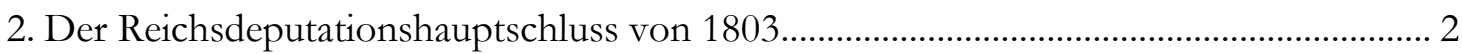

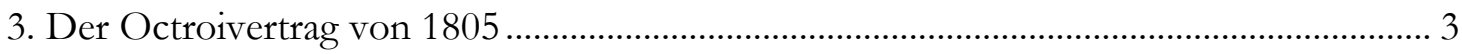

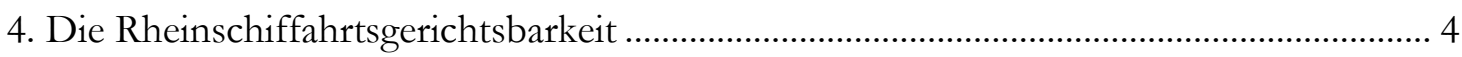

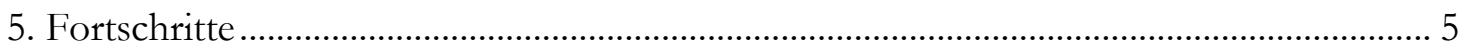

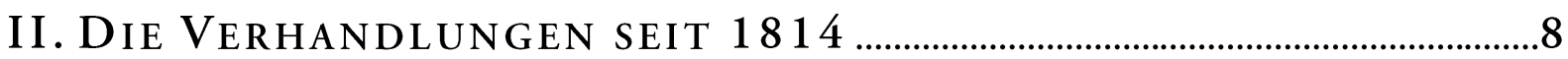

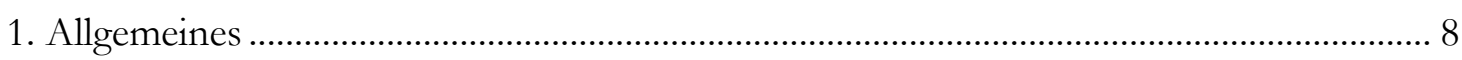

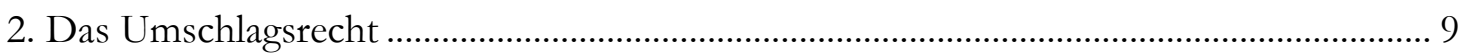

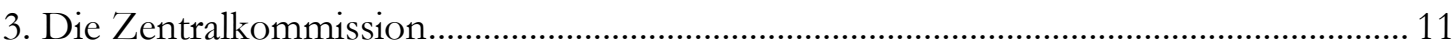

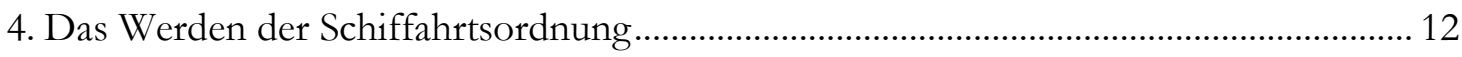

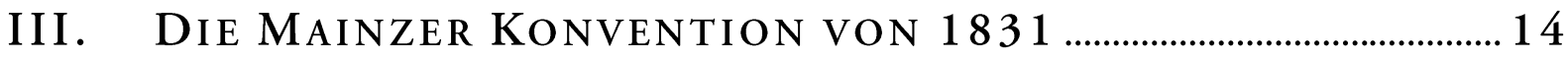

1. Der Inhalt der Mainzer Akte ................................................................................................. 14

2. Das Verhalten der Schweiz ................................................................................................ 15

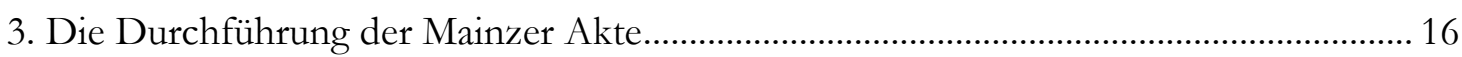

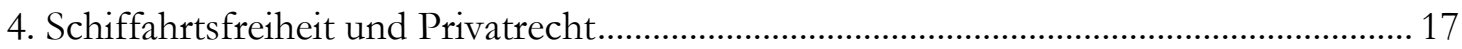

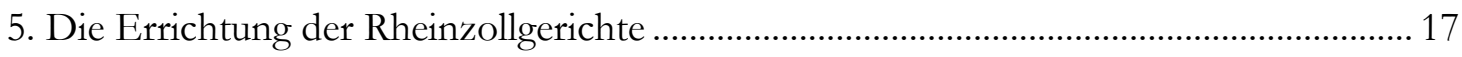

IV. Die Entwicklung bis Zur Mannheimer AkTe $1868 \ldots \ldots \ldots \ldots . . . . . .18$

1.Die Ablösung der freiwilligen Rangfahrten ........................................................................... 18

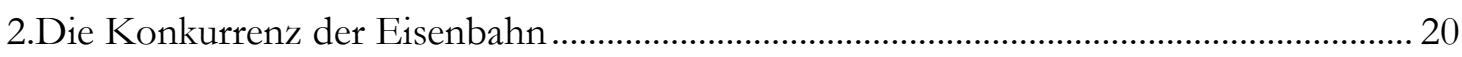

3.Änderungen der Mainzer Akte ....................................................................................... 20

4.Die Revidierte Rheinschiffahrtsakte von 1868.................................................................... 21

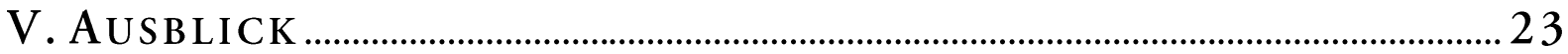

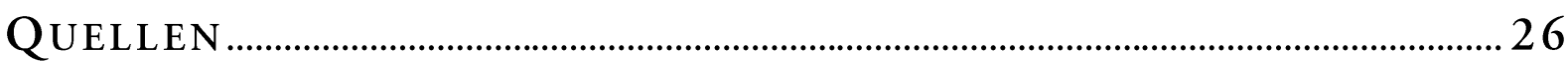

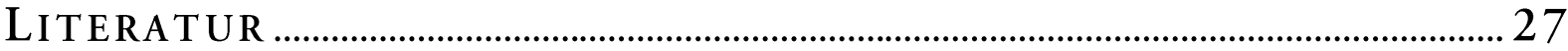




\section{Kurzer Blick in die Vorgeschichte}

\section{Allgemeines}

Im Frieden von Campoformio (17. Oktober 1797) hatte Österreich nicht nur Belgien und Venetien an Frankreich übertragen, sondern auch der Abtretung der deutschen Länder auf dem linken Rheinufer an Frankreich zugestimmt. Über die Entschädigung der deutschen Fürsten, die dadurch ihre Gebiete verloren, verhandelte der Rastatter Kongress zwischen 1797 und 1799 ergebnislos. Die Reichsdeputation regte zwar mehrfach an, die Rheinschiffahrt allgemein freizugeben, die französischen Unterhändler wollten jedoch freie Schiffahrt nur Frankreich und Deutschland zugestehen, alle anderen Länder sollten nur dann daran teilhaben, wenn diese beiden Staaten zustimmten und die Bedingungen für die Teilnahme stellen durften ${ }^{1}$. Die bisherigen Beschränkungen der Schiffahrt bis ans Meer, also alle Zölle, Stapel- und Umschlagrechte, wollten die französischen Unterhändler aufgehoben wissen, doch drangen sie bei der Reichsfriedensdeputation damit nicht durch, die geltend machte, der Uferbau werde dadurch unbezahlbar ${ }^{2}$. Immerhin wurde dort die grundsätzliche Freiheit der Rheinschiffahrt angeregt ${ }^{3}$. Der Art. VI des Friedens von Lunéville vom 9. Februar $1801^{4}$ schlug - völkerrechtlich verbindlich - das linksrheinische Deutschland zu Frankreich und machte den Talweg des Rheines ${ }^{5}$ zur Grenze beider Staaten ${ }^{6}$. Frankreich war auf diese Weise Rheinanlieger geworden.

\section{Der Reichsdeputationshauptschluss von 1803}

Es waren noch die Verhandlungen zweier weiterer Jahre nötig, um die Rheinschiffahrt neu zu regeln. Das Ergebnis zeigt $\$ 39$ des Reichsdeputationshauptschlusses vom 25. Februar $1803^{7}$, es war im Wesentlichen das Folgende:

1 Eberhard Gothein, Entwicklung S. 30, der auf die Protokolle der Session 29 v. 6. März 1798 und die französische Note vom 3. Mai 1798, in den Beilagen zu den Protokollen V, S. 5 verweist.

2 HEINRICH BERNHARD OPPENHEIM, S. 79 mit weiteren Nachweisen.

3 Eberhard Gothein, S. 33.

4 Friedensvertrag v. Lunéville v. 9. Febr. 1801, anerkannt vom Deutschen Kaiser, Wien, am 27. Febr., vom deutschen Reichstag durch Reichsgutachten vom 12. März (vgl. Reichsgutachten v. 7. März 1801), Ratifikation durch Kaiserliches Commissions-Dekret v. 9. März 1801 und den Reichsschluss vom 10. Mai 1801. Der erste französischen Konsul anerkannte ihn am 11. März 1801(PHILIPP ANTON GUIDO V. MEYER/HEINRICH ZOEPFL, 1. Theil, S. 4); vgl. FrIEDRICH Wilhelm GHillany, I, S. 287 ff; KARl Zeumer, Nr. 211, S. 508; HANS Hubert Hofmann, Nr. 65 b, 2, c, S. $326 \mathrm{ff}$.

5 Über den Talweg vgl. HeINRICH BERNHARD OPPENHEIM, S. 81 m. Fn. 5, der auf JEAN BAPTISTE-MOISE JOLLIVET, Du Thalweg du Rhin, considéré comme limite entre la France e l'Allemagne, an X, übersetzt in: „Betrachtungen über den Thalweg des Rheins in Hinsicht als Grenzscheide zwischen Frankreich und Deutschland; über die auf beiden Rheinufern angelegten Zölle, Mauthen und die den zwei Städten Mainz und Kölln zustehende Stapelgerechtigkeit", Mayence (A. Crass), Vendémiaire an X (1802) bei CHRISTIAN ECKERT, S. 379f; vgl. EBERHARD GOTHEIN, S. 33.

$6 \quad$ Alfred Lederle, S. 74.

7 Bei Karl ZEUMER Nr. 212, S. 509 - 528, hier: S. 521. 
1. Alle bisherigen Zölle waren aufgehoben und durften nicht wieder errichtet werden.

2. Stattdessen wurden Eingangsgebühren (droits de douane) und ein Schiffahrts-Octroi (Schiffssteuer) von Frankreich und dem deutschen Reich gemeinsam erhoben.

3. Der Kurfürst-Erzkanzler [Fürstprimas v. Dalberg] wurde ermächtigt, für das Reich den Inhalt dieses Octroi auszuhandeln.

4. Die Taxe des Octroi durfte die bisherigen Zölle nicht übersteigen.

5. Zur Erhebung wurde eine besondere Behörde errichtet, deren Generaldirektor Frankreich und der Kurfürst-Erzkanzler gemeinsam ernannten; die Abgabeneinnehmer des rechten Rheinufers ernannte der Kurfürst-Erzkanzler im Einvernehmen mit den Landesfürsten.

6. Es wurden mindestens fünf und nicht mehr als fünfzehn Erhebungsbureaux errichtet.

7. Aus dem Ertrag des Octroi wurden zunächst die Kosten der Erhebung, der Verwaltung und der Polizei bestritten; der Überschuß in zwei gleiche Teile geteilt, von denen der eine dem Unterhalt der Leinpfade (chemins de halage) ${ }^{8}$ und der Ufer dienen sollte, der andere dazu bestimmt war, die Dotation des Kurfürst-Erzkanzlers und der in $\$ \$ 9,14,17,19$, u. 20 gegebenen Anweisungen - unter anderem der in $\$ \$ 7$ und 27 subsidiarisch und bedingnißweise angewiesenen Renten zu erwirtschaften. Ein etwaiger Überschuß sollte die Lasten des Schiffahrtsoctroi ablösen.

Eine französische Grenzregulierungskommission legte den Talweg des Rheines fest. Als maßgeblichen Zeitpunkt für diese Grenzziehung bestimmte Frankreich am 18. März 1805 den Zeitpunkt, an dem die Ratifikationsurkunden des Lunéviller Friedens ausgewechselt wurden?. „Talweg“ war nicht etwa der Treidelpfad (der zwischen beiden Ufern wechselte), sondern der auslegungsbedürftige Name für den Flusslauf selbst. Die Festlegung des Talweges war und blieb wegen der häufigen Verlagerung des Rheinbettes höchst unklar, auch erlaubte dieser Begriff die Zugehörigkeit von Rheininseln zu Frankreich oder Deutschland willkürlich zu handhaben. Die Kommission beließ es bei ihrer Arbeit nicht beim Talweg, sondern legte eine Talwegachse ${ }^{10}$ fest, die künftig die Grenze bildete. Infolgedessen schlug sie Rheininseln (z. B. die Petersau bei Mainz, die Ingelheimer und Eltviller Aue) zu Frankreich. Dahinter stand Frankreichs Streben, den Rhein gleichsam als „Festungsgraben"11 zu betrachten. Nach Abschluss des Rheinbundes (12. Juli 1806) ${ }^{12}$ gliederte Napoleon - im Widerspruch zu seiner Note vom 1. Aug. $1806^{13}$ - die Brückenköpfe Kehl, Kassel bei Mainz, Kostheim und die rechtsrheinisch gelegene Petersinsel Frankreich an, nachdem vorher schon die rechtsrheinische Festung Wesel Frankreich zugeschlagen worden war. Er wollte sich damit gegen das Reich militärisch absichern. Auf Widerstand stieß er nicht: Das Reich und seine Fürsten erkannten diese Grenzziehung stillschweigend $\mathrm{an}^{14}$.

\section{Der Octroivertrag von 1805}

Zuvor war aber am 15. August 1804 der Octroivertrag zwischen Frankreich und dem

8 Über Leinpfade vgl. DieTer WerkMÜLLER, Art. Leinpfad, in: HRG, Bd. II, Sp. 1831 - 1837.

9 Frieden von Lunéville v. 9. Februar 1801 vgl. oben 4).

10 Vgl. JOHANN LUDWIG KLÜBER, Staatsrecht, $\$ 60$.

11 So: EBERHARD GOTHEIN, S. 29.

12 Bei Karl Zeumer, Nr. 214, S. 532 - 536 = Hans Hubert HOFMANN Nr. 69 (Auszug), S. 374 $-392$.

13 Text bei Heinrich Bernhard OPPENHEIM, S. 90.

14 Heinrich Bernhard OPPENHEIM S. 85 ff. 
Deutschen Reich geschlossen worden ${ }^{15}$. Allein Frankreich und der Kurerzkanzler $v$. Dalberg hatten ihn ausgearbeitet und Preußen dabei umgangen ${ }^{16}$. Er war ein völkerrechtlicher Vertrag mit 132 Artikeln und regelte nicht nur die internationalen Verhältnisse, sondern enthielt auch staatsrechtliche und polizeiliche Vorschriften. Der Rhein sollte fortan ein gemeinschaftlicher Fluss Frankreichs und Deutschlands sein, der einheitlich verwaltet werden sollte. Die Zentralverwaltung mit Sitz in Mainz war neutral und von den Anliegerstaaten unabhängig. Sie bestand aus einem Generaldirektor und vier Inspektoren (Artt. 45; 49; 54, 56 des Octroivertrages), die dort Sitz und Stimme hatten. Sie hatten die Erhaltung des Flussbettes und der Leinpfade zu beaufsichtigen, die Einnahmen der Erhebungsbüros zu überwachen, die Register abzuschließen und die Kassen zu prüfen. Ihnen zugeordnet waren Unterbeamte ${ }^{17}$, welche die Abgaben erhoben. Ihrer Bestechlichkeit sollte Art. 83 vorbeugen.

Die Zentralverwaltung war zum einen Verwaltungsbehörde, welche die Gebühren zu erheben und gemäß der Konvention zu verteilen hatte, sie hatte zum anderen auch richterliche Aufgaben und war schließlich auch gesetzgeberisch tätig, weil sie Ergänzungs- und Ausführungsbestimmungen zur Konvention erlassen konnte (Art. 130).

\section{Die Rheinschiffahrtsgerichtsbarkeit}

Schiffer, welche den Octroi übertraten (z. B. Art. 117, Octroibetrug), wurden bestraft. Sie sollten aber möglichst ohne lange Unterbrechung ihrer Fahrt abgeurteilt werden. Im Bericht des ersten Generaldirektors, Johann Joseph Eichhoff ${ }^{18}$, für die Wiener Flusskommission von 1815, der auf dem Octroi beruht ${ }^{19}$, ist der Instanzenzug dargestellt. Danach waren die Octroieinnehmer (les receveurs) die Richter erster Instanz (veritables juges, Art. 122). Sie konnten Beweise vor Ort erheben und die Schiffsbesatzung sofort vernehmen. Die Kontrolleure (contrôlleurs) übten das Amt eines Staatsanwaltes aus und die Beseher (visiteurs de douane) vertraten die Interessen des Fiskus (les agents de fisce). Da die Octroibüros nach Art. 46 über den ganzen Strom verteilt waren ${ }^{20}$, stellte man damit eine schnelle Bearbeitung der Übertretungen sicher. Die auf Geldstrafe lautenden Urteile durften

15 Der Octroi beruht auf dem vom Kaiser am 27. April 1803 ratifizierten Reichsdeputationshauptschluss und der von beiden Vertragspartnern am 11. Mai 1805 (am 27. Thermidor an XII) in Paris ratifizierten Fassung, mit Zusatzartikel vom 1. Oktober 1804. Er trat am 1. Nov. 1805 in Kraft; Druck in: RHEINURKUNDEN I, Nr. 4, S. 6 - 25, auch bei PHILIPP ANTON GUIDO V. MEYER, Staatsakten I, S. 45 - 65, vgl. CHRISTIAN ECKERT, S. 19 ff.

16 Eberhard Gothein, S. 36.

17 Von ihnen handeln die Artt. 47; 50 - 52; 55f; 64; 67 - 71; 84; 87; 89.

18 JOHANN JOSEPH EICHHOFF war der Koch des letzten kurkölnischen Kurfürsten MAXIMILIAN FRANZ V. ÖSTERREICH $(1784-1801)$ gewesen. Da er lange zur Ausbildung in Paris gelebt hatte und gut Französisch sprach, war er zum Generaldirektor des Rheinschiffahrtsoctroi ernannt worden, vgl. EBERHARD GOTHEIN, S. 119.

19 JOHANN JOSEPH EICHHOFF, in: Rheinurkunden, I, Nr. 42, S. 106.

20 Nach Art. 46, des Octroi waren linksrheinische Octroibüros errichtet in Neuburg, Mainz, Andernach, Köln, Homberg, Griethausen (später: Lobith), rechtsrheinische in Mannheim, Wellmich (später: Kaub), Thal - Ehrenbreitstein (später: Koblenz), Linz, Düsseldorf und Wesel, vgl. RHEINURKUNDEN I, Nr. 4, S. 12. Die Zusatzartikel vom 1. Oktober 1804 verlegten das Octroibüro von Griethausen nach Lobith, von Thal-Ehrenbreitstein nach Koblenz und von Wellmich nach Kaub, vgl RHEINURKUNDEN I, Nr. 4, S. $25 \mathrm{f}$. 
provisorisch vollzogen werden (Art. 122). Das Verfahren war kostenfrei (Art. 126), auch durften Schiff und Ladung nicht mit Arrest belegt werden ${ }^{21}$.

Die Zentralverwaltung bildete die zweite richterliche Instanz, dort konnte man gegen Urteile der ersten Instanz Rekurs einlegen. Dieses Rechtsmittel hatte keine aufschiebende Wirkung, die Octroieinnehmer konnten ihre Entscheidungen stets provisorisch vollziehen. Als Gericht bestand die Zentralverwaltung aus dem Generaldirektor und zweien seiner Inspektoren; sie entschied mit Stimmenmehrheit ihrer Mitglieder und tagte in Mainz (Art. 122). Ob es sich dabei um ein ordentliches Gericht handelte, ist streitig, weil es eigentlich ein Verwaltungsstrafverfahren war. Um aber seine Einheitlichkeit und eine schnelle Entscheidung zu gewährleisten, hatte der Octroivertrag dieses Verwaltungsverfahren einer Gerichtsbarkeit stark angeglichen (rechtliches Gehör, Zeugenvernehmung, Urteilsbegründung) so daß ein gerichtliches Verfahren letztlich zu bejahen ist ${ }^{22}$.

Gegen Entscheidungen der zweiten Instanz konnte man nach Art. 123 Rekurs bei einer dritten Instanz einlegen. Auch sie bestand aus drei Personen, die sich im Brumaire (Okt./Nov.) jeden Jahres in Mainz zur Rechtsprechung versammelten, nämlich dem Präfekten des Départements Donnersberg als Vertreter Frankreichs, einem vom Kurerzkanzler zu ernennenden Kommissar als Vertreter Deutschlands und einem deutschen oder französischen Rechtsgelehrten (jurisconsulte), auf den sich die Kommissare einigen mußten. Auch diese Kommission entschied mit Stimmenmehrheit ${ }^{23}$. Eine Rechtsmittelfrist bestand nicht, und das Verfahren war schriftlich auf Grund der Aktenlage und der Schriftsätze der Parteien, Art. 124. Da diese Kommission nur Recht sprach, handelt es sich zweifelsfrei um ein ordentliches Gericht.

\section{Fortschritte}

Der Octroivertrag von 1805 brachte gegenüber dem früheren Zustand einige Fortschritte: Der Einfluss der kleinen Uferstaaten auf die zu leistenden Abgaben war aufgehoben, denn die neue Zentralverwaltung mit ihrer hierarchischen Ordnung überwachte die gesamte Schiffahrt von der Schweiz bis zur Grenze der Batavischen Republik, es bestand also eine einheitliche Verwaltung ${ }^{24}$. An die Stelle der vielen Rheinzölle der Anrainer (die zuletzt 32 Erhebungsstellen verzeichnete) war eine Octroiabgabe getreten, die nur noch an zwölf Stellen des Rheines erhoben wurde. Ein voller Betrag lag nur noch auf Handelsware (marchandises), während nach den Artt. $102-105^{25}$ solche Güter, die Ackerbau und Industrie förderten ${ }^{26}$ oder den Hausbedarf der Anwohner deckten, nur mit einem geringeren Tarif belastet waren, der häufig nur $1 / 4$ oder $1 / 20$ des normalen Tarifs ausmachte ${ }^{27}$. Auch die Personenbeförderung ${ }^{28}$ war mit einem ermäßigten Tarif belegt. Bezahlt wurde der Octroi

21 DiETER KISCHEL, Geschichte, S. 14.

22 Vgl. Friedrich Wilhelm Gaum, Bemerkungen, S. 106f; dagegen DiETER KisCHEL, Geschichte, S. $15 \mathrm{f} ; 19 \mathrm{ff}$.

23 Johann Joseph Eichhoff, in: Rheinurkunden, I, Nr. 42, S. 106, vgl. Dieter KISCHEL, Geschichte, S. $17 f$.

24 Heinrich Bernhard Oppenheim, S. 93.

25 RHEINURKUNDEN I, Nr. 4, S. 21.

26 Art. 1102 spricht von l'aventage de l'agriculture et de l'industrie des pays riverains.

27 Heinrich Bernhard Oppenheim, S. 94; Christian Eckert, S. 22.

28 Sogenannte Yachten und Wasserdiligencen, Art. 101: Les yachts, diligences d'eau ... destiné au transport de voyageurs...payeront ... du quart de marchandises qu'ils pourroient embarquer en raison 
auf dem linken Rheinufer in Francs, auf dem rechten in deutscher Konventionsmünze, deren Kurs auf Dauer festgelegt war (Art. 107).

Diese Octroigelder waren eine Steuer, die den Verkehr auf dem Strome nach wie vor belasteten; sie hatten mit dem Eingangszoll für Waren (droits de douanes) nichts zu tun. Aber sie hatten einen festen Tarif, der niedriger war als vorher, sie wurden schnell und bequem erhoben, und es gab keine Mehrforderungen, weil Art. $83^{29}$ der Bestechlichkeit und Willkür der Einnehmer wirksam vorbeugte. Unter dem Strich sanken die Einnahmen aus dem Octroi gegenüber den früheren Zöllen auf die Hälfte, und die Schiffer wurden entsprechend geringer belastet ${ }^{30}$. Außerdem floss der Großteil der Gebühren in den Unterhalt des Stromes und der Leinpfade (chemins de halage) ${ }^{31}$, so daß sich die Verhältnisse auf dem Rhein erheblich besserten.

Da man sich auf dem Rastatter Kongress $\left(1797\right.$ - 1799) ${ }^{32}$ über die Abschaffung des Stapels in Mainz und Köln, der meist als Umschlagszwang und Staffelrecht oder Stationsfahrt (relâche et échelle) ausgeübt wurde, nicht hatte einigen können ${ }^{33}$, so war er sowohl im Reichsdeputationshauptschluss von 1803 als auch im Rheinoctroi von 1805 bestehen geblieben. Umschlagszwang war die Pflicht, Handelswaren, die an einer Stadt (z. B. Mainz oder Köln) vorbeigeführt werden sollten, dort auszuladen und von Fuhrleuten oder Schiffern dieser Stadt weiterbefördern zu lassen. Es handelt sich also um ein Transportmonopol städtischer Schiffer, das häufig mit einem Speditionsmonopol städtischer Kaufleute verbunden war $^{34}$. Damit wollte man nicht nur Mainz und Köln begünstigen, vielmehr sprachen fiskalische Gründe für die Beibehaltung des Stapels, weil so der Eingang von Mauten und Gebühren leichter überwacht und sog. ,Heckenfahrten' und ,Winkelspeditionen“ verhindert werden konnten ${ }^{35}$, die den Eingangszoll zu umgehen suchten. Köln blieb auch

de leurs tonnage“.

29 RHEINURKUNDEN I, Nr. 4, S. 16.

30 Damit entsprach der Tarif auch dem Art. 39 des Reichsdeputationshauptschlusses (RHEINURKUNDEN I, Nr. 1, S. 4), wonach die Rheinschiffahrtsgebühren die alten Zölle nicht übersteigen durften, vgl. CHRISTIAN ECKERT, S. 24, m. Fn. 2.

31 Art. 33 - 36 des Octroi und Art. 44 (Aufgaben der Kontrolleure), RheINURKUNDEN I, Nr. 4, S. $11 \mathrm{f}$.

32 Nachdem Österreich im Frieden von Campoformio (17. Okt. 1797) der Abtretung des linken Rheinufers an Frankreich zugestimmt hatte, sollten die betroffenen Fürsten in Deutschland entschädigt werden. Der Rastatter Kongress1797 - 1799 konnte sich jedoch darüber nicht einigen; erst der Reichsdeputationshauptschluß vom 25. Febr. 1803 (bei KARL ZeUMER, Nr. 212, S. 509 - 528, brachte eine Lösung.

331804 hatte der berühmte rheinische Jurist HEINRICH GOTTFRIED WILHELM DANIELS, der sich auf dem Titel seines Gutachtens als „Rechtsgelehrter und Professor bei der Centralschule in Kölln" nannte, in einem Gutachten für die Kölner Handelskammer den Erhalt des Stapelrechts in Köln und Mainz befürwortet. Diese Auftragsarbeit gipfelt in dem Satz: „Das Stapelrecht ist das einzige Mittel für das linke Rheinufer, die unschätzbaren Vortheile zu erhalten, welche der Kommissions- und Transithandel darbietet. Er ist von höchster Wichtigkeit für die Republik [Française], obschon es der Staatskasse derselben keinen direkten Geldzufluß verschaft", vgl. Daniels, S. 42.

34 Vgl. OTto GÖNNENWEIN, Stapel, S. 96 ff; 143 ff; KARL OTTO SCHERNER, Rheinakten S. 585.

35 Vgl. Johann Joseph EICHHOFF, S. 130f; NAU, Beiträge II, S. 78; zitiert bei CHRISTIAN ECKERT, S. 25f; vgl. MATTHIEU SCHWANN, S. 206-221; 236- 241. 
nach 1804 Umladeplatz (Stationshafen) der Güter, die aus Niederlanden kamen, Mainz war Umladeplatz für Güter, die zwischen Köln und Straßburg befördert wurden.

Immerhin gab es einige Verbesserungen: So brauchten die Handelswaren in Mainz und Köln nicht mehr angeboten zu werden, auch durften auf Grund des Umschlagsrechts keine Gebühren mehr erhoben werden; ausgenommen waren nur Kran-, Kai- und Wägegebühren, für die jedoch Maximalbeträge festgesetzt wurden, Artt. 8, 9 ${ }^{36}$. Auch galt das Mainzer Umschlagsrecht nicht für Waren, die (etwa von Köln) mit Mainzer Schiffen und Schiffern für Frankfurt/M bestimmt waren: Sie durften unmittelbar dorthin gebracht werden, ebenso wie Waren aus dem Oberrhein, wenn im Mainzer Hafen die Schiffahrtsgebühren bezahlt wurden. Das alte Frankfurter Recht, ohne Umschlag den Rhein zu Tal zu fahren, war stillschweigend aufgehoben ${ }^{37}$. Im Übrigen behielt Frankfurt seine Messefreiheiten (franchises des foires, Art. 10 - 12).

Im Rheinland hatte Frankreich die alten Schifferzünfte zwar bereits 1798 aufgelöst $^{38}$, um die Freiheit der Schiffahrt zu gewährleisten, die Octroikonvention sah aber in den Artt. $14-16^{39}$ vor, daß in den Stationshäfen Schiffervereine zu gründen seien. Neu war, daß bei diesen Vereinen die lokale Begrenzung fehlte: Jeder Schiffer konnte ihnen nach Art. 15 beitreten, wenn er die fachliche Eignung nachwies, also ein gedienter Steuermann mit einem Patent war. Die Schiffer erhielten ihre Frachtaufträge nach gesetzlicher Ordnung (Art. 18) ${ }^{40}$, der sogenannten Rangfahrt ${ }^{41}$ und bildeten in der Folge Gilden, die lediglich der Aufsicht der Zentralbehörde unterstanden ${ }^{42}$.

Auch die Frachthöhe war nicht frei vereinbar. Die Octroiverwaltung mußte nach Art. 13 der Konvention von einer Frankfurter Messe zur nächsten (alle halbe Jahr) Gutachten der Handelskammern von Köln, Mainz und Straßburg und der Magistrate von Düsseldorf, Frankfurt und Mannheim einholen und danach die Frachtpreise festlegen, die Höchstpreise waren ${ }^{43}$. Dies galt nicht für die Markt- und Personenschiffahrt, welche die Yachteigner mit sog. Wasserdiligencen ${ }^{44}$ und Postschiffen betrieben und nicht für die Fähren, die häufig nur Nachen waren. Denn die Artt. 19 - 23 des Octroi hatten sie weder an Stationen noch an Schiffervereine gebunden ${ }^{45}$.

36 RHEINURKUNDEN I, Nr. 4, S.7.

37 Vgl. dazu HEINRICH BERNHARD OPPENHEIM, S. 97.

38 Vgl. STEINS, S. 42; KARL OTTO SCHERNER, Rheinakten S. 586.

39 Bereits am 21. Germinal an XIII (11. April 1805) ordnete Frankreich die Errichtung solcher Gilden an, die jetzt keine städtischen Zünfte mehr waren, sondern der Generaldirektion unterstanden. Die Mainzer und die Kölner Handelskammer hatten Schifferordnungen durchgesetzt; allerdings haben die Generaldirektion und das französische Ministerium sie stark umgearbeitet. Erst am 12. August 1807 ist die neue Schifferordnung veröffentlicht worden, vgl. EBERHARD GOTHEIN, S. $45-49$.

40 HEINRICH BERNHARD OPPENHEIM, S. 97; CHRISTIAN ECKERT, $27 f$.

41 Rangfahrt (tour de rôle) hieß, daß der reisende Kaufmann keinen beliebigen Schiffer mit der Fracht beauftragen konnte, sondern daß er den nehmen mußte, der als nächster an der Reihe war (wie heute noch bei Taxifahrten), vgl. KARL OTTO SCHERNER, Rheinakten, S. 585.

42 Die Gildeordnung für Schiffer trat in Mainz am 12. August 1807 in Kraft (HEINRICH HERMAN, S. 96 - 101; vgl. HEINRICH BERNHARD OPPENHEIM, S. 98 (der fälschlich 1806 angibt), kurz darauf in Köln (HEINRICH HERMAN S. 110 - 128); ausführlich: dazu: CHRISTIAN ECKERT, 31 ff; zur Aufsicht: S. 38; EBERHARD GOTHEIN S. 45 ff; MATTHIEU SCHWANN S. 271.

43 Vgl. CHRISTIAN ECKERT, S. 45 - 57.

44 Von diligence, Emsigkeit, Eile, also: Eilschiffahrt.

45 Vgl. Art. 19 - 23 der Octroikonvention (RHEInURKunden I, Nr. 4, S. 9f). Dagegen sollte der 


\section{Die Verhandlungen seit 1814}

\section{Allgemeines}

Der Rheinbund, bestehend aus Frankreich und den deutschen Satelliten Napoleons, hatte sich im Jahre 1814 stillschweigend aufgelöst ${ }^{46}$, und der erste Pariser Friede vom 30. Mai 1814 versprach in Art. 6: „Les états de l'Allemagne seront indépendans et liés par un lien fédératif". Der Freiherr vom Stein als Vorsitzender der Zentralverwaltung der Verbündeten zog im Herbst 1813 den bisherigen Generaldirektor des Octroi, Johann Joseph Eichhoff, nach Wien und übergab die Rheinschiffahrtssachen dem Grafen Solms-Laubach, der „im Namen der hohen alliirten Mächte" eine vorläufige Verwaltung nach der Octroikonvention ('administration provisoire de l'octroi du Rhin) von 1805 einrichtete, und 1815 aus sachlichen Gründen einige Erhebungsbüros verlegte, so z. B. das Büro von Homberg nach Ruhrort $^{47}$ - wegen der Kohleschiffahrt von der Ruhr in den Rhein, die von Homberg aus nicht zu kontrollieren war.

Während Frankreich in den Friedensverhandlungen verlangte, der Rhein solle die Grenze zu Frankreich bleiben, lehnten die Verbündeten das für den mittleren Teil des Stromes ab. Für den Oberrhein blieb zwar die alte Talwegsgrenze bestehen ${ }^{48}$, doch ordnete der erste Pariser Frieden die 1801 Frankreich einverleibten linksrheinischen Gebiete wieder Deutschland zu; sie wurden auf dem Wiener Kongress Preußen, bzw. Bayern und Österreich zugeschlagen. Hier wurde der Rhein wieder zum deutschen Strom.

Art. 5 des ersten Pariser Friedens bestimmte, daß die Schiffahrt auf dem Strome frei sein sollte ${ }^{49}$. Einzelheiten waren auf dem in Aussicht genommenen Kongress der Verbündeten festzulegen. Bereits am 14. Dezember 1814 ernannte das Komitee der Hauptmächte einen Ausschuß mit dem Auftrag, auf Grund des Art. 5 und der $\$ \$ 2$ und 3 des geheimen Artikels III des ersten Pariser Friedens, die Einzelheiten zu auszuhandeln. Der Kommission sollten ursprünglich nur vier Mitglieder (Österreich, Preußen, England und Frankreich) angehören, doch erweiterte man sie um die Niederlande, Bayern, Baden, Hes-

Verkehr zwischen Frankfurt und Mainz nach Art. 24 durch eine Wasserdiligence mit Namen „Marktschiff" von Frankfurter und Mainzer Schiffern gemeinsam durchgeführt werden, vgl. CHRISTIAN ECKERT, S. $58 \mathrm{ff}$.

46 Vgl. JOHANN LUDWIG KLÜBER, Öffentliches Recht d. deutschen Bundes, $\mathbb{\$}$ 47; HEINRICH BERNHARD OPPENHEIM, S. 101.

47 Dieses Vorgehen hat WilHELM V. HumbOLDT gegenüber der Klage Straßburgs gerechtfertigt, vgl. JOHANN LUDWIG KLÜBER, Akten, Bd. III, S. 56 ff; 155 - 160; vgl. HEINRICH BERNHARD OPPENHEIM, S. 126, Fn. 1; EBERHARD GOTHEIN, S. $64 f$.

48 Erster Pariser Friede, Art. 3, Punkt 5 (bei Philipp AnTon Guido V. MEYER, Staatsakten, I, S. 241); vgl. HEINRICH BERNHARD OPPENHEIM, S. 108.

49 Art. 5 lautet: „La navigation sur le Rhin, du point où il devient navigable jusqu'à la mer et reciproquément, sera libre de telle sorte qu'elle ne puisse être interdite à personne, et l'on s'occupera au futur congrès de principes d'après lesquels on pourra régler les droits à lever par les états riverains, de la manière la plus égale et la plus favorable au commerce de toutes les nations ...". (HEINRICH HERMAN, Gesetze, S. 537); vgl. CHRISTIAN ECKERT, S. 80. Damit war der Rechtszustand wiederhergestellt, der im römischen Recht (Inst. 2. 1. 1, 4; Dig. 39. 2. 24) und im Mittelalter (trotz des Königseigentums und der Regalrechte) bis ins 15./16. Jahrhundert gegolten hatte, vgl. OTTO GÖNNENWEIN, Freiheit, S. $12 \mathrm{f}$. 
sen-Darmstadt und Nassau ${ }^{50}$, zu denen später noch Württemberg und Kurhessen als Rheinuferstaaten traten. Die Verhandlungen sollten den Vorschlägen folgen, die Wilhelm v. Humboldt in seinem Gutachten „Mémoire préparatoire sur le travail de la Commission de navigation" vom 3. Februar 1815 zusammengestellt hatte ${ }^{51}$. Er forderte zunächst, die Grundsätze festzulegen, die den Interessen des Handels dienten, diese dann auf die Verhältnisse des Rheins und der Schelde (l'Escaut) anzuwenden und deren Besonderheiten beizufügen, schließlich die gleichen Grundsätze auf die anderen Flüsse Europas zu übertragen. Für den Rhein sollten nicht nur die seit 1814 gegenüber dem Octroi von 1805 veränderten territorialen Verhältnisse, sondern auch die Rentenzahlungen entsprechend dem geplanten Deutschen Bund überprüft werden.

Den Beratungen wurde jedoch der vom französischen Vertreter Fürstprimas v. Dalberg vorgelegte Entwurf zugrunde gelegt, der sich vornehmlich am Octroi orientierte ${ }^{52}$, aber die Ergebnisse des Pariser Friedens berücksichtigen musste. In Art. 12 seines Entwurfs schlug er nicht nur eine gemeinsame Erhebung des Octroi, sondern auch seine Verteilung proportional den Uferstrecken des schiffbaren Rheins vor. Das wäre für Frankreich außerordentlich günstig gewesen, da es am Oberrhein nur geringe Gebühren einnahm, weil von Basel nur wenige Schiffe rheinab, aber gar keine rheinauf fahren konnten ${ }^{53}$. Deshalb folgte die Kommission diesem Vorschlag nicht.

\section{Das Umschlagsrecht}

Einer der ersten großen Streitpunkte war das Stapel- und Umschlagsrecht (droit de relâche) von Köln und Mainz. Es war schon auf dem Rastatter Kongress (1797/99) heftig umstritten gewesen, doch hatte der Octroi 1805 das Umschlagsrecht aufrechterhalten und nur die Anbietungspflicht der Waren beseitigt ${ }^{54}$. Allerdings hatte der französische Generaldirektor der Brücken und Landstraßen in Köln durch Verordnung betr. die Herabmilderung des Umschlagszwanges vom 7. November $1809^{55}$ ihn für eine Reihe von Massengütern - darunter Kohle - abgeschafft. Das machten sich Franz Haniel und die Brüder Stinnes zu nutze, indem sie Kohlen von der Ruhr nicht nur nach Holland, sondern den Rhein hinauf als Kleinschiffer transportierten und verkauften. Da sie nicht Mitglied einer Rangfahrt auf einer Stromstrecke waren ${ }^{56}$, brauchten sie sich um die Vorschriften der Rangfahrten nicht zu kümmern.

50 Beschluß der Kongreßkommission vom 2. Februar 1815, bei JOHANN LUDWIG KLÜBER, Akten, Heft 9, S. 11 ff.

51 Druck des Gutachtens bei JoHANn Ludwig KLÜBER, Akten, Bd. III, S. 24 - 30.

52 Projet d'articles pour le règlement de ce qui concerne la navigation, des grands fleuves traversant plusieurs territoires, présenté par M. le duc de Dalberg, plénipotentiaire de France bei JOHANN LUDWIG KLÜBER, Akten, Bd. III, S. $13-19$.

53 Vgl. Heinrich Bernhard OPPENHEIM, S. 113.

54 Siehe oben I. 5.

55 Verordnung des französischen Generaldirektors der Brücken und Landstraßen vom 7. Nov. 1809, in: Rhein.-Westfäl. Wirtschaftsarchiv (RWWA)1, 23b, 19 F 63/64, zitiert bei JÜRGEN HEINZ SCHAWACHT, S. 84, Fn. 469. 1815 wurden weitere Massengüter befreit: „Töpfer- und Pfeifenerde, Gips, Stahl, Plattsteine, Schieferstein, Erdenware, Erdentöpfe, Laubholz, Bretter, Tuffstein und Traß, leere Fässer, Schleifstein und loses Steingut aller Art" (RWWA 1, 23b, 19 F 61/62 (29. 3. 15), zitiert bei JÜRGEN HEINZ SCHAWACHT, S. 84, Fn. 468.

56 Vgl. EBERHARD GOTHEIN, S. 119. MATHIAS STINNES versuchte 1818 auch - zusammen mit der 
Auf dem Wiener Kongress taten sich Köln und Mainz zusammen, um ihre Rechte zu verteidigen ${ }^{57}$. Eine Fülle von Druckschriften der Verteidiger und der Gegner des Stapels $e^{e r s c h i e n}{ }^{58}$, ohne daß neue Sachargumente aufgetaucht wären. Die Kommission lud Vertreter der Verteidiger und der Gegner vor und hörte ihre Argumente an $^{59}$, beschloss dann jedoch einmütig, den Stapel abzuschaffen ${ }^{60}$. Das entsprach dem wachsenden wirtschaftlichen Liberalismus der Zeit, der die vielen Schutzvorschriften der Vergangenheit zunehmend als Zwang empfand und folgte damit den Gedanken Adam Smiths $s^{61}$ über den Wohlstand der Nationen, die der englische Vertreter in der Kommission, Richard le Poer Trench, zweiter Lord of Clancarty, vehement zu vertreten wußte. Die Regierungen haben diesen Beschluß der Kommission alsbald gutgeheißen.

Da Art. 108 der Wiener Kongressakte die Vertragschließenden nur verpflichtete, untereinander Verträge über die Gestalt der Schiffahrtsfreiheit auf dem Rhein zu schließen ${ }^{62}$, verschwand das Umschlagsrecht in Köln und Mainz keineswegs sofort nach dem 9. Juni 1815, vielmehr schleppte es sich noch 15 Jahre fort. Die Kölner konnten nämlich die Differenzen zwischen den Niederlanden und Preußen wegen der niederländischen Zollerhebung nutzen, um es zu erhalten: Es diente gewissermaßen als Druckmittel, um die niederländische Zollerhebung zu beseitigen ${ }^{63}$. Immerhin hatte ein Erlass des Kölner Oberbürgermeisters von 1818 den Umschlagszwang weiter erleichtert ${ }^{64}$. Erst die Rheinschiffahrtsakte vom 31. März 1831 hat in ihren Artikeln 43 und $44^{65}$ das Umschlagsrecht und die

niederländischen Firma SNOECK, BRESSER \& BALK aus Arnheim - eine neue Beurtfahrt zwischen Arnheim und Köln bzw. Mülheim/Rhein zu organisieren, stieß dabei aber auf den Widerstand der Rotterdamer Beurtschiffer, so daß sie 1820 verboten wurde; vgl. JÜRGEN HEINZ SCHAWACHT, S. 87, der auf RWWA 1, 40, 5 F 260/261 und auf PIETER JAN BOUMAN, Untergang, S. 247 verweist.

57 Vgl. oben Fn. 55. Die Verordnung vom 24. August 1818, betr. Abänderungen für den Umschlag und die Erhebungen im Hafen von Köln (bei HEINRICH HERMAN, S. 284 ff), hob den Umschlagszwang für deutsches Getreide, Salinensalz, Eisenwaren, Mineralwasser etc. (S. 285), weil die Umladungskosten - gemessen am Wert der Waren - dem Handel untragbar schienen. Dagegen hielt Mainz am Umschlagsrecht fest, vgl. CHRISTIAN ECKERT, S. 117 ff; EBERHARD GOTHHEIN, S. 119.

58 Vgl. die Darstellung bei CHRISTIAN ECKERT, S. 82 - 89; MATTHIEU SCHWANN, S. 206 - 221; $236-241$.

59 Vgl. JOHANn LUDWIG KLÜBER, Akten, Bd. III, S. 33 - 71; vgl. CHristian ECKERT, S. 86 ff; MATTHIEU SCHWANN S. 366 - 389.

60 Beschluss in der Sitzung vom 23. Februar 1815 bei JOHANN LUDwIG KLÜBER, Akten, III, S. 59, vgl. EBERHARD GOTHEIN, S. 77; CHRISTIAN ECKERT, S. 88f.

61 Vgl. ADAM SMITH' († 17. Juli 1790) Werk „An inquiry into the nature and causes of the wealth of nations", das 1776 erschienen war.

62 Art. 108 der Wiener Kongreßakte vom 9. Juni 1815 bei JOHANN LUDWIG KLÜBER, Quellen, S. 92; vgl. OTTO GÖNNENWEIN, Freiheit, S. 8.

63 Vgl. Heinz WASSERMEYER, S. $294 \mathrm{ff}$; Clemens v. Looz-Corswarem, S. $336 \mathrm{ff}$

64 Die Verordnung vom 24. August 1818 betr. Abänderungen für den Umschlag und die Erhebungen im Hafen von Köln befreite deutsches Getreide und Salinensalz, Schleifsteine, Rübkuchen, Wacholderbeeren, rohes Gußeisen, altes Eisen, gegossenes Eisen, Töpfe, Öfen, Mineralwasser, Nüsse, leere Fässer und Reifen vom Umschlag (bei HEINRICH HERMAN, I, S. 284 - 286); vgl. CHRISTIAN ECKERT, S. 117; EberHARd GOTHEIN, S. 119; JÜrGEN HEINZ SCHAWACHT, S. 89, Fn. 502.

65 Druck: RHEINURKUNDEN I, Nr. 80, S. 242 
Schiffergilden endgültig aufgehoben ${ }^{66}$. Das trug Köln und Mainz herben finanziellen Verlust ein. Während aber der Preußische Staat die Stadt Köln entschädigte, erhielt Mainz keinerlei Verlustausgleich ${ }^{67}$.

\section{Die Zentralkommission}

Ein weiterer Streitpunkt waren die zukünftigen Befugnisse der Zentralkommission ${ }^{68}$. Man hielt sie zwar allgemein für notwendig, folgte aber Preußen nicht, das eine starke Zentralbehörde wünschte und vorschlug, daß sich die Zahl ihrer Mitglieder nach dem Verhältnis der Besitzungen der einzelnen Staaten am Rheinufer richten sollte. Die kleinen Anliegerstaaten wollten aber für ihren Stromanteil die Landeshoheit nur im kleinstmöglichen Rahmen eingeschränkt wissen ${ }^{69}$ und die Abgaben im eigenen Interesse und durch eigene Beamte erheben. Die Vorschläge Wilhelm v. Humboldts vom 24. Februar $1815^{70}$ für eine starke Kommission fanden infolgedessen nicht den Beifall der kleinen Staaten. Deshalb ließ der französische Vorschlag vom 24. Februar 1815 den Gedanken einer starken übernationalen Behörde mit ausschließlicher Zuständigkeit für den gesamten Rheinlauf fal$\operatorname{len}^{71}$. Sie sollte nur ein Mal im Jahr (erforderlichenfalls ein zweites Mal) ${ }^{72}$ tagen, und man maß ihr nur beratende Funktion (un caractère consultatif) $\mathrm{zu}^{73}$. Nach einer Intervention Wilhelm v. Humboldts wurde jedoch in der 8. Sitzung am 14. März 1815 ein revidierter Text beschlossen, welcher die ständigen Aufseher (Inspektoren) wieder einführte und ihnen die Überwachung des künftigen Vertrages zuwies ${ }^{74}$. In der 12. Sitzung vom 24. März 1815 wurde die Flußschiffahrt endgültig geordnet und die Bevollmächtigten der acht größeren Mächte unterzeichneten die Beschlüsse ${ }^{75}$. In dieser Form wurden sie Bestandteil der Artt. 108 - 117 über die Rheinschiffahrt der Wiener Kongressakte vom 9. Juni $1815^{76}$.

66 Vgl. die Darstellung bei HEINRICH BERNHARD OPPENHEIM, S. 128 und ausführlich bei CHRISTIAN ECKERT, S. $117-124$.

67 Preußen gewährte Köln im Laufe der Jahre insgesamt 232.000,-- Taler Entschädigung, vgl. CHRISTIAN ECKERT, S. 231.

68 Verhandelt in der 5. Sitzung, vgl. JOHANn LUDWIG KLÜBER, Akten III, S. 82 ff; vgl. HeINRICH BERNHARD OPPENHEIM, S. 116.

69 Vgl. JOHANNES BÄRMANN, S. 17 ff; ausführlich: VITÁNYI, S. 56 ff.

70 Gedruckt in: RHEINURKUNDEN I, Nr. 42, S. 92 - 95.

71 HEINRICH BERNHARD OPPENHEIM, S. 116f; vgl. den Text des Vorschlages in: RHEINURKUNDEN, I, Nr. 42, S. $86 \mathrm{ff}$.

72 So der letzte Vorschlag Wilhelm V. HumboldTS vom 14. März 1815 (RheinurKunden I, Nr. 42, S. 145, Art. 11).

73 Protokoll v. 24. Februar 1815, RHEINURKUNDEN I, Nr. 42, S. 86, Beschluss Nr. 534.

74 Zu diesen Verhandlungen vgl. WILLEM JAN MARIE VAN EYSINGA, Zentralkommission, S. $13 \mathrm{ff}$.

75 Druck in PHILIPP ANTON GUIDO V. MEYERs Staatsakten, S. 202 ff; 208 - 219; und in RHEINURKUNDEN I, Nr. 42, S. 158 ff, wo für den Rhein auf den Annex Nr. 16 B der Wiener Schlußakte (S. $43 \mathrm{ff}$ ) verwiesen ist; vgl. HeINRICH BERNHARD OPPENHEIM, S. 119, mit Fn. 3; ChristiAN ECKERT, S. $89 \mathrm{ff}$.

76 Druck in: RheINURKUnden I, Nr. 41, S. 42 - 50, zugleich Artt. 108 - 117 der Wiener Kongressakte (bei JOHANN LUDWIG KLÜBER, Quellensammlung, S. 92 - 95) mit dem Annex Nr. 16 B aus JOHANN LUDWIG KLÜBER, Acten Bd. III, S. 257 - 275 (= Akten der Wiener Flußkommission); auch (in deutscher Übersetzung) bei WILLEM JAN MARIE VAN EYSINGA, Zentralkommission, Anhang II, S. 139 - 146. 
Danach war die Schiffahrt auf dem Rhein frei, der Bodensee gehörte zwar nicht dazu, aber die Strecke zwischen Basel ${ }^{77}$ und Straßburg sowie der niederländische Rhein bis zu seinen Mündungen war eingeschlossen. Die Abgaben, Zölle und die Schiffergebühr blieben etwa denen des Octroi gleich, auch die zwölf Erhebungsbüros blieben bestehen und jeder Staat erhob die Steuern auf eigene Rechnung. Die Zentralkommission kontrollierte die Durchführung des Reglements. Alle Umschlagsrechte, Schifferprivilegien und alle Abgaben außer den genannten blieben aufgehoben, aber es waren noch viele Einzelheiten zu regeln, namentlich die Fracht- und Octroitaxen, die Schiffergesellschaften, die Organisation der Gerichte, die Unterhaltung des Flussbettes und der Treidelpfade. Die nach Art. 30 des Reichsdeputationshauptschlusses v. 25. Febr. 1803 zu zahlenden Octroi-Renten übernahmen die Uferstaaten gegenüber Frankreich, das sich 1810 die Rentenansprüche des Fürstprimas Dalberg hatte abtreten lassen $^{78}$.

\section{Das Werden der Schiffahrtsordnung}

Die 1813 eingesetzte provisorische Rheinschiffahrtsdirektion sollte eigentlich am 1. Juni 1815 ihre Tätigkeit beenden, die Zentralkommission und eine endgültige Ordnung der Rheinschiffahrt eingeführt werden ${ }^{79}$, doch verlängerte man die Frist bis zum Jahre $1817^{80}$. Im Jahre 1816 setzten - entsprechend den Beschlüssen in der Wiener Kongreßakte - die Uferstaaten Frankreich, Baden, Bayern, Hessen, Nassau, Preußen und die Niederlande die neue Zentralkommission ein. Sie löste die provisorische Octroiverwaltung ab, die seit Herbst 1813 bestanden hatte ${ }^{81}$. Diese Kommission sollte vor allem eine Schiffahrtsordnung für den Rhein erarbeiten. Das erwies sich als eine langwierige und mühselige Kleinarbeit, die nur schleppend vorankam: Die Verhandlungen über die Schiffahrtsordnung auf dem Rhein dauerten 15 Jahre und durchliefen vier Abschnitte:

Der erste Abschnitt dauerte 5 Jahre, nämlich von der Konstituierung der Kommission am 5. August 1816 bis zum September 1821: Man bemühte sich, eine interimistische Instruktion zu verabschieden, brachte viele Entwürfe ein, konnte sich aber nicht einigen. Da ein Ende der Verhandlungen nicht abzusehen war, legte Preußen am 7. September 1821 den Entwurf einer endgültigen Rheinschiffahrtsordnung vor ${ }^{82}$.

77 Zur fehlenden Teilhabe der Schweiz an der Rheinschiffahrtsakte vgl. WilHELM ALBERT CHIESA, S. 89 ff; KARL OTTO SCHERNER, Begründung, S. 68; vgl. unten III. 2.

78 Der Anteil des Fürstprimas v. DALBERG an den nach Art. 39 des Reichsdeputationshauptschlusses zu erhebenden und zu verteilenden Octroi-Renten war die Entschädigung nach Art. 25 ebenda für seine 1801 an Frankreich gefallenen linksrheinischen Gebiete. Die Verpflichtung der Rheinuferstaaten zur Zahlung der Rente beruht auf Art. 28, N. 1 des Annexes Nr. 16 B zu Art. 117 der Wiener Kongressakte vom 9. Juni 1815 (Druck: RHEINURKUNDEN I, Nr. 41, S. 48) und auf $\$ 30$ des Reichsdeputationshauptschlusses von 1803 (bei KARL ZEUMER, Nr. 212, S. 516f; 518; 521f); vgl. HEINRICH BERNHARD OPPENHEIM, S. 123f.

79 Vgl. die Note Wilhelm v. HumboldTs vom 7. April 1815, in: Johann Ludwig KLÜBERs Akten Bd. III, S. 275 ff (276); HeINRICH BERNHARD OPPENHEIM, S. 127, Fn. 3.

80 Vgl. den Entwurf dieses Beschlusses bei JOHANN LUDWIG KLÜBER, Akten III, S. $277 \mathrm{ff.}$

81 Vgl. JOHANN LUDWIG KLÜBER, Öff. Recht, $\$ 570$, Fn. b), S. 848.

82 Vorgelegt in der 225. Sitzung vom 7. September 1821, vgl. JOHANNES BÄRMANN, S. $17 f$. 
(2) Die Verhandlungen über diesen Entwurf bildeten den $\boldsymbol{z}$ weiten $\boldsymbol{A} \boldsymbol{b} \boldsymbol{s} \boldsymbol{c h n i t \boldsymbol { t }}$, sie dauerten bis zum 13./15. Juli 182483, also fast 3 Jahre.

Von 1824 an datiert der dritte Abschnitt, denn es ergaben sich Schwierigkeiten zwischen Preußen und den Niederlanden. Die Kommission unterbrach deshalb die Beratungen über die endgültige Ordnung und empfahl zweiseitige Verhandlungen zwischen diesen beiden Staaten. Sie drehten sich vornehmlich um die Auslegung von Art. $5^{84}$ des ersten Pariser Friedens. Dort heißt es: „La navigation sur le Rhin, du point où il devient navigable jusqu'à la mer et réciproquement, sera libre...", und um die ganz ähnliche lautende Formulierung in Art. I des Annexes 16 B zu Art. 117 der Wiener Kongressakte ${ }^{85}$ : Es ging um das Verständnis der Worte „libre jusqu'a la mer“. Hieß das frei bis ans Meer oder bis ins Meer (jusque dans la pleine mer)? Während Preußen meinte, es sei gemeint "jusq'a la pleine mer ${ }^{86}$ (bis zum offenen Meer), also freie Schiffahrt durch Lek und Waal bis ins Meer, bezogen die Niederlande diese Worte nur auf den sog. konventionellen Rhein zwischen Straßburg und Lobith. Ihrer Meinung nach war der kaum befahrene Lek die eigentliche Verlängerung des Rheines, der vielbefahrene Waal aber ein „besonderer Fluss“ und nicht zum Rhein gehörig, auf ihn sei die Wiener Akte deshalb nicht anzuwenden. Da die Stromstrecke von Lobith bis zum Meer bereits Ebbe und Flut unterlag, war sie holländischer Ansicht nach zudem nicht fluvial (flussgebunden) und zählte für sie bereits zum Meer, sei also von diesen Artikeln nicht betroffen ${ }^{87}$. Infolgedessen hatten die Niederlande nach Abschluss des Wiener Kongresses ihre alten Abgaben und Zölle dort wiedereingeführt $^{88}$. Sie füllten damit ihre Kassen, lähmten aber den freien Schiffsverkehr auf dem Rhein $^{89}$.

Die Folge war ein 16-jähriger Wirtschaftskrieg zwischen Preußen und den Niederlanden, der erst 1830 beigelegt wurde ${ }^{90}$. In den zweiseitigen Verhandlungen zwischen beiden Staaten lenkten nämlich die Niederlande erst ein, nachdem am 24. August 1830 in Belgien ein Aufstand ausgebrochen war, der am 4. Oktober 1830 in der belgischen Unabhängigkeitserklärung mündete ${ }^{91}$. Die Niederlande fürchteten, Belgien werde in der Zollfrage

83 Es handelte sich um die 327. Sitzung vom 13./15. Juli 1824, vgl. JOHANNES BÄRMANN, S. 18; KARL OTTO SCHERNER, Rheinakten S. 587.

84 Erster Pariser Friede, Art. 5 (bei Philipp Anton Guido V. MeYer, Staatsakten, I, S. 241), der vollständige Text des Artikels findet sich oben in Fn. 49, vgl. HEINRICH HERMAN, Gesetze, S. 537; CHRISTIAN ECKERT, S. 80.

85 RHEINURKUNDEN I, Nr. 41, S. 43.

86 Verhandlungen vom 27. August 1825, vgl. JOHANNES BÄRMANN, S. $18 f$.

87 Vgl. CHRISTIAN ECKERT, S. 101; v. EYSINGA S. 25 ff. Außerdem erklärte Holland den kaum befahrenen Lek zur eigentlichen Verlängerung des Rheines (RICHARD HENNIG S. 85).

88 Es handelte sich um Ein- und Ausfuhrzoll, um einen Beitrag zu den Kriegskosten, Plombier- und Passgebühren, Hafen- und Wägegelder. Auch verbot Holland den Transit von Gewürzen (Zimt, Muskat, Nelken), Tee, und Papier mit holländischem Wasserzeichen, vgl. ausführlich: JOHANN LUDWIG KLÜBER, Öff. Recht $\$ 571$, Fn. d), S. 850 - 853; HEINRICH BERNHARD OPPENHEIM, S. 134.

89 Vgl. ausführlich: JOHANN LUDWIG KLÜBER, Öff. Recht, $\$$ 571, Fn. d), S. 850 - 853; NAUS Beiträge, Bd. II, S. 87; HEINRICH BERNHARD OPPENHEIM, S. 127.

90 Vgl. KARL GRIEWANK, S. 232.

91 Die Unabhängigkeit Belgiens als selbständigen Staat garantierte der „Traité pour la séparation de la Belgique, d'avec la Hollande, signé à Londres"zwischen Österreich, Belgien, Frankreich, Großbritannien, Rußland und Preußen vom 15. Nov. 1831, Druck bei GEORGES FRÉDÉRIC DE MAR- 
nachgeben und den Verkehr von den niederländischen Rheinmündungsarmen fort auf die südlichen Flussläufe lenken ${ }^{92}$. Deshalb schlossen sie mit Preußen einen Kompromiss und beide Staaten legten der Zentralkommission einen geänderten Entwurf für eine Rheinschiffahrtsordnung vor.

Damit begann der vierte Abschnitt der Verhandlungen, der zur Grundlage der Mainzer Rheinschiffahrtsakte von 1831 wurde. Da man sich über die richtige Auslegung des Passus jusqu'a la mer nicht einigen konnte, beschloss man, sich auf die technisch-administrative Seite des Schiffsverkehrs zu beschränken und die Freiheit des Handels und des Schiffahrts-Gewerbes nicht zu regeln ${ }^{93}$.

Während der 15-jährigen Verhandlungen hatten sich - teils wegen der Teilaufhebung des Kölner Umschlagszwangs teils wegen der zwischenzeitlichen technischen Entwicklung - die Verhältnisse auf dem Rhein stark verändert. Zwar betrieben die Schiffergilden noch immer den Transport der eigentlichen Handelswaren und hielten auch an den Rangfahrten fest, doch hatten sich die Kleinschiffer der Verfrachtung der umschlagfreien Landesprodukte, vor allem der Ruhrkohle, bemächtigt ${ }^{94}$ und versorgten damit den Mittel- und Niederrhein. Außerdem entstanden seit den 1820er Jahren Dampfschiffahrtsgesellschaften am Rhein. Diese Gesellschaften schlossen sich bald zusammen und nahmen den traditionellen Schiffern mehr und mehr die Frachten und die Existenzmöglichkeit ab, ohne daß dem abgeholfen werden konnte ${ }^{95}$.

\section{Die Mainzer Konvention von 1831}

\section{Der Inhalt der Mainzer Akte}

Die Mainzer Akte datiert vom 31. März $1831^{96}$. Sie war ein völkerrechtlicher Vertrag, der durch Austausch der Ratifikationsurkunden am 16. Juni 1831 für die Unterzeichner verbindlich wurde. Ihr Text wurde in den Regierungsblättern der beteiligten Staaten verkün$\operatorname{det}^{97}$. Dies genügte damals, um völkerrechtliche Normen zu Staatsgesetzen zu machen ${ }^{98}$.

TENS, Nouveau Recueil Général Bd. 11, S. 390; vgl. JÜRGEN HEINZ SCHAWACHT, S. 21 ff; CLEMENS v. LOOZ-CORSWAREM S. 337.

92 Vgl. RiCHARD HENNIG, S. 85.

93 So zuerst im 467. Protokoll der Zentralkommission vom 19. August 1829. Der Vertrag sollte beschränkt werden auf: „les mesures, et les dispositions réglementaires, dont la navigation du Rhin ne peut se passer pour longtemps, sur la base d'un ensemble de propositions faites et acceptées réciproquement, sous la réserve expresse toute fois, que cet accord ne portera aucun préjudice aux droits et aux principes soutenus de part et d'autre" (zitiert bei JOHANNES BÄRMANN, S. 19f, bestätigt im 495. Protokoll vom 28. Sept. 1830.

94 Vgl. CHRISTIAN ECKERT, S. 143 ff; KARL OTTO SCHERNER, Rheinakten S. 587.

95 Vgl. KARL OTTO SCHERNER, Begründung, S. 70.

96 Text bei GEORGES FRÉDÉRIC DE MARTENS, Nouveau Recueil Bd. IX, S. 252; in der G S 1831, S. $71 \mathrm{ff}$ u. in RHEINURKUNDEN I, Nr. 80, S. 212 - 280; vgl. LARS U. SCHOLL, S. $22 \mathrm{ff}$.

97 So im Preußischen Regierungsblatt 1831, S. 71 ff; im Regierungsblatt f. d. Königreich Bayern 1831, S. $384 \mathrm{ff}$.

98 Denn die dualistische Theorie, wonach Völkerrecht durch ein Transformationsgesetz in staatliches Recht umgewandelt werden mußte, entstand erst gegen Ende des 19. Jhs., vgl. AlFrED VERDross, Völkerrecht, S. 117 f; KARL OTTO SCHERNER, Rheinakten S. 591. 
Der Vertrag enthielt zehn Titel:

Der 1. Titel (Artt. 1 - 13) enthielt die Zugeständnisse, welche die Niederlande und Preußen sich gegenseitig und gegenüber allen Vertragsstaaten hinsichtlich der Fahrt von Lobith bis zum Meer durch Lek und Waal, die zur Verlängerung des Rheins erklärten (Art. 2), sowie die allgemeine Zollfreiheit der Rheinschiffahrt. Alle Schiffe sollten künftig nur einer festbestimmten Abgabe (droit fixe) unterworfen sein (Art. 4);

der 2. Titel (Artt. 14 - 35) behandelte die Rheinschiffahrtsabgaben;

der 3. Titel (Artt. 36 - 41) regelte die Anwendung der einzelstaatlichen Steuergesetze auf die Rheinschiffahrt;

der 4. Titel (Artt. 42 - 47) behandelte die Fachten und Rangfahrten; wobei wichtig ist, daß Art. 43 nun endgültig das Umschlagsrecht abschaffte; Art. 44 löste die Schiffergilden und Zünfte auf ${ }^{99}$, und Art. 45 ließ die Zahl der Rheinschiffer unbestimmt und regelte in Art. 42 nur die nötigen Fachkenntnisse für die Ausübung der Rheinschiffahrt; wofür sie das Schifferpatent verlangte. Für die Fährschiffahrt und für Fahrten innerhalb eines Staates galt die Mainzer Akte überhaupt nicht (Art. 46);

der 5. Titel (Artt. 48 - 52) ließ zwar noch Rangfahrten zu, macht aber die Beteiligung an derartigen Verträgen freiwillig;

der 6. Titel (Artt. 53 - 70) enthielt die Polizeivorschriften zur Sicherheit der Rheinschiffahrt (Lotsen, Tauglichkeit der Schiffe, Haftung des Spediteurs und des Schiffers, Instandhaltung der Leinpfade).

der 7. Titel (Artt. 71 - 80) behandelte die Folgen der Nichtzahlung von Schiffahrtsabgaben;

der 8. Titel (Artt. 81 - 88) sah Rheinschiffahrtsgerichte, (damals: Rheinzollgerichte, vgl. Art. 8109) vor und machte sie nicht nur für Abgabenstreit und Strafsachen, sondern auch für deliktische Zivilsachen zuständig (Ersatz des durch die Schiffahrt fahrlässig angerichteten Schadens, Art. 81, d);

der 9. Titel (Artt. 89 - 108) regelte die Amtsbefugnisse der Zentralkommission und der vier Aufseher und der 10. Titel (Art. 109) den Vollzug des Vertrages.

\section{Das Verhalten der Schweiz}

Die Schweiz war übrigens weder an den Verhandlungen in Wien noch an der Rheinschiffahrtsakte beteiligt, obwohl diese von Basel ab gelten sollte. Sie war damals zu stark mit ihren inneren Angelegenheiten beschäftigt ${ }^{101}$, als dass sie sich um die Wiener Verhandlungen hätte kümmern wollen, doch suchte die Schweizer Republik gleichwohl die Vorteile zu nutzen, welche ihr die Mainzer Akte bot. So erkannten die Rheinanlieger 1841 die Baseler Schiffseichungsvorschriften als verbindlich an. Seit 1852 näherte sich die Schweiz den übrigen Rheinanliegerstaaten und schloss Verträge über die Schiffahrt auf dem Bodensee und dem Hochrhein bis Basel, ohne jedoch Mitglied der Zentralkommission zu werden ${ }^{102} .1855$ fragte die Zentralkommission in Basel an, ob die Schweiz die neuen Regeln über die Schifferpatente für ihre Schiffer gelten lassen wolle ${ }^{103}$. So hat sich die

99 RHEINURKUNDEN, I, Nr. 80, S. 242.

100 RHEINURKUNDEN, I, Nr. 80, S. 257.

101 Über die Schweiz als Rheinuferstaat aus schweizerischer Sicht vgl. RUdOLF BAUMGARTNER, S. $46 f$.

102 Vgl. Wilhelm Albert CHIESA, S. 92 ff.

103 Vgl. James VAlOTTON, S. 287f; Wilhelm Albert CHIESA, S. 90. Am 9. Dez. 1848 erhielt die 
Schweiz allmählich dem internationalen Rhein-Regime genähert, ohne doch die Mitgliedschaft zu erwerben ${ }^{104}$, die sie erst 1919 erhielt.

\section{Die Durchführung der Mainzer Akte}

Im Gegensatz zur Wiener Kongressakte verschwand nach der Ratifizierung der Mainzer Akte das Umschlagsrecht sofort. Bereits am 17. Juli 1831 wurden die letzten Reste des Stapelrechts in Köln und Mainz beseitigt ${ }^{105}$, mit der Folge, daß der Rhein nun eine gemeinschaftliche Handelsstraße war, auf der kein Schiff mehr zu unfreiwilligem Aufenthalte oder zur Umladung gezwungen werden durfte.

Art. 10 der Akte sah die Einrichtung von acht Freihäfen vor (in Preußen: Köln und Düsseldorf; in Hessen: Mainz, später wurde ihre Zahl auf 19 vermehrt) ${ }^{106}$, es waren Niederlagsplätze für fremde Waren, ohne dass diese für die Niederlage, das Ein- und Ausladen mit Zoll belegt werden durften.

Die Schiffergilden kämpften um ihren Fortbestand. So versuchten die Mainzer, sich die Ladungen nach Köln zu erhalten, doch konnte ihnen der Mainzer Magistrat nicht helfen, da fortan alle Verbindungen zu den Kaufleuten und Spediteuren auf freier Vereinbarung beruhten. So lösten sich die Gilden nach dem in Art. 44 der Mainzer Akte vorgesehen Verfahren selbst auf, die Mainzer im Jahre $1833^{107}$. An ihre Stelle trat zeitweilig die Vereinbarung freiwilliger Rangfahrten, sog. Beurten ${ }^{108}$, welche die Artt. 49 - 51 der Akte nach niederländischem Vorbild zwischen Handelsstädten und einzelnen Schiffern zugelassen hatten ${ }^{109}$. Sie bewährten sich zunächst, weil sie regelmäßige und schnelle Transportverbindungen boten. Bereits vor Verabschiedung der Mainzer Akte hatten sich die Handelskammern einiger Städte um solche Verträge bemüht. Köln wollte sich zur Zentrale des Rheinverkehrs machen. Dort und in Mainz versuchte man, so den Verlust des Umschlagsrechtes auszugleichen ${ }^{110}$. Mainz wurde dabei aber bald von Mannheim überholt, das einen direkten Verkehr zwischen Ober- und Niederrhein einrichtete, so zwischen Mannheim und Köln, Amsterdam und Rotterdam. Köln blieb der Knotenpunkt der Fahrten nach Mainz, Mannheim und Frankfurt sowie nach den Niederlanden.

Rheinschiffahrtsakte v. 1831 den Supplementar-Artikel XIX über die Schifferpatente (RHEINURKUNDEN I, Nr. 240, S. 495 ff); es folgte am 24. August 1852 eine abändernde Vereinbarung der Zentralkommission (erwähnt in RHEINURKUNDEN I, Nr. 264, S. 534).

104 Erst der Versailler Frieden von 1919 gewährte der Schweiz die volle Mitgliedschaft in der Zentralkommission als Rheinuferstaat im nouveau régime international du Rhin, vgl. WILHELM ALBERT CHIESA, S. $105 \mathrm{ff}$.

105 Vgl. CHRISTIAN ECKERT, S. 231.

106 Vgl. CHRISTIAN ECKERT, S. 231.

107 Vgl. CHRISTIAN ECKERT, S. $232 \mathrm{ff}$.

108 „Beurt“ (niederl., heißt Reihe, Ordnung, „an de Beurt liggen“ an der Reihe sein zu fahren; „Beurtfahrt" meint also nichts anderes als Rangfahrt). Vgl. CHRISTIAN ECKERT, S. 225; $244 \mathrm{ff}$, der auf eine Verordnung für die Rheinschiffahrtskommission zu Amsterdam vom 15. April 1825 bei HEINRICH HERMAN, Adressbuch 1827, S. 47 - 64 hinweist.

109 Dazu ausführlich: JÜRGEN HEINZ SCHAWACHT, S. 82 ff.

110 Vgl. CHRistian ECKERT, S. 246 ff; EBERHARD GOTHEIN, S. 202 ff; KARL OTTO SCHERNER, Rheinakten, S. 592. 
Hinzuweisen ist auch darauf, dass Preußen in Ergänzung der Mainzer Akte im Haager Vertrag vom 5. November $1842^{111}$ und im Antwerpener Vertrag vom 20. Mai $1843^{112}$ die freie Schiffahrt auch auf den Flüssen und Kanälen zwischen Rhein und Schelde vereinbarte.

\section{Schiffahrtsfreiheit und Privatrecht}

Neben der Beseitigung von Umschlagsrecht und verpflichtender Rangfahrt enthält die Mainzer Akte vor allem öffentlich-rechtliche Regelungen, um die Sicherheit des Schiffsverkehrs zu gewährleisten. Dazu gehören Normen über den Zugang zum Schifferberuf ${ }^{13}$, die Sicherheit der Schiffe, ungehinderte Durchfahrt und die Sicherung des Leinpfades. Zivilrechtlich waren die Rheinzollgerichte allerdings nicht für Vertragsverletzungen zuständig, sondern nur für Ansprüche Dritter aus unerlaubter fahrlässiger Verletzung ihrer Rechte durch den Schiffsverkehr. Die Gerichte sahen solche unerlaubten Handlungen allein in der Verletzung schiffahrtspolizeilicher Verhaltenspflichten ${ }^{114}$.

Dagegen schützte die Mainzer Akte nicht die Freiheit des Handels, sie betraf deshalb auch nicht Export- und Importbeschränkungen einzelner Staaten. Der 1833 gegründete Deutsche Zollverein, dem unter preußischer Führung die meisten deutschen Staaten unter Ausschluss Österreichs beitraten ${ }^{115}$, förderte die Handelsfreiheit in Deutschland ungemein. Privatrecht fand sich nur in Art. 48 der Mainzer Akte, der die Freiheit garantierte, Frachtverträge zu frei vereinbarten Frachtraten abzuschließen, also die verpflichtende Rangfahrt abschaffte. Sonstige privatrechtliche Regelungen sollten lediglich der Sicherheit der Schiffahrt dienen, sie verwiesen im Übrigen nur auf das geltende Recht einzelner Staaten. Nach Art. 53, Absätze III, IV sollte der Spediteur für Schäden haften, die infolge der Untauglichkeit des Schiffes an der Fracht eingetreten waren. Diese Regelung sollte ihn darauf hinweisen, sich vor Abschluß des Frachtvertrages das Untersuchungszeugnis des Schiffes vorlegen zu lassen, und so zur Sicherheit des Verkehrs beizutragen.

\section{Die Errichtung der Rheinzollgerichte}

Um die Gerichte unabhängig zu machen, hatte die Mainzer Akte in ihrem 8. Titel eine Prozeßordnung für die Rheinzollgerichte erster Instanz geschaffen. Damit ihr Verfahren zügig lief, war es summarisch (Art. 84) und fand in erster Instanz vor dem Einzelrichter statt, der unbeschränkt für Fiskal- und Strafsachen zuständig war. In Zivilsachen durfte er jedoch nur über unerlaubte Handlungen richten. In der zweiten Instanz gab es nur ein Gericht für jeden Staat (Art. 87). Die Akte verpflichtete die Rheinanliegerstaaten - unabhängig von ihrer Gerichtsverfassung - auf eine einheitliche Gerichtsbarkeit für den ganzen Rhein, wie sie schon der Wiener Kongressgeplant hatte ${ }^{116}$. Allerdings fehlte in der

111 Vgl. RICHARD HENNIG, S. 86.

112 Vgl. RiCHARD HENNIG, S. 86.

113 Normen über das Prüfungswesen für Rheinschiffer erließ z. B. Preußen mit dem „Regulativ wegen Ausübung der Rheinschiffahrt von diesseitigen Untertanen und wegen des Lootsendienstes auf dem Rhei$n e$ "vom 5. August 1834, G S 1834, S. 149 = RHEINURKUNDEN I, Nr. 107, S. $314-321$.

114 Vgl. , J. B. EMIL v. TRAUT, S. 77 ff; KARL OTTO SCHERNER, Rheinakten, S. 592.

115 Über die vielschichtige Entstehung dieses Zollvereins und seiner Vorgänger vgl. GERHARD LINGELBACH, Art. Zollverein, in: HRG, Bd. V (1996), Sp. 1769 ff.

116 Vgl. DIETER KISCHEL, Geschichte, S. 49- 63. 
Akte ein Schutz des Beklagten (rechtliches Gehör, freie Verteidigung, öffentliche und mündliche Verhandlung in Strafsachen).

In Ausführung der Mainzer Akte errichteten die Anrainerstaaten pünktlich und gleichförmig die Gerichte ${ }^{117}$, indem sie zwischen 1831 und 1834 Ausführungsbestimmungen erließen und die Richter auf die Akte von 1831 vereidigten. In Preußen geschah das durch die Verordnung vom 30. Juni $1834^{118}$. Sie bestimmte in den $\$ \$ 1$ und 2 für die preußische Rheinstrecke 21 Rheinzollgerichte erster Instanz, errichtete jedoch keine neuen Gerichte, sondern machte - entgegen dem Sinn des Art. 81 - eine Anzahl rheinpreußischer Untergerichte (zumeist die Friedensgerichte) für Rheinschiffahrtssachen zuständig ${ }^{119}$. Als Berufungsgericht benannte die VO in $\$ 4$ den dritten Zivilsenat des Rheinischen Appellationsgerichtshofes in Köln, zuständig für Zivil-, Fiskal- und Strafsachen ${ }^{120}$.

\section{Die Entwicklung bis zur Mannheimer Akte 1868}

\section{Die Ablösung der freiwilligen Rangfabrten}

Die Beurtfahrten bedienten sich rheinab meist der Segel, rheinauf des Leinzuges. Um jeweils frische Pferde zu haben ${ }^{121}$ und damit ihre Schnelligkeit zu erhöhen, gründete man Relaistationen. Gleichwohl waren die Dampfschlepper mit angehängten eisernen Kähnen, die seit den 1820er Jahren auf dem Rhein auftauchten und bald die gesamte Stromstrecke von Holland bis Straßburg befuhren, für die alten Rangfahrer eine gefährliche Konkurrenz, weil sie viel geringere Frachtpreise anbieten konnten ${ }^{122}$ und dazu noch schneller fuhren. Das zeigen die Fahrtzeiten: So brauchte ein Rangfahrer, der von Amsterdam bis Köln segelte und getreidelt wurde, zu einer Bergreise 14 bis 30 Tage, von Rotterdam 10 bis 20 Tage. Dagegen benötigten die Dampfer und Dampfschlepper mit ihren Kähnen von Amsterdam bis Köln 5 bis 8 Tage, von Rotterdam 3 bis 5 Tage $^{123}$. Traten zunächst hol-

117 Vgl. den Abdruck der Errichtungsverordnungen für die Rheinzoll- (=Rheinschiffahrts-) gerichte in RHEINURKUNDEN I, Nr. 83 (Niederlande: Gesetz v. 9. Juli 1831); Nr. 84; 92 (Baden: VO v. 15. Juli 1831); Nr. 86; 88 (Hessen: VO v. 5. Oktober 1831); Nr. 91 (Frankreich: Gesetz v. 21. April 1832); Bayern: durch Reskript v. 9. März 1833 (nicht in RHEINURKUNDEN); Nr. 105 (Preußen: VO 30. Juni 1834 [G S 1834, S. 136], vgl. die Ergänzung in Nr. 106 [G S 1834, S. 145]).

118 Verordnung wegen Einrichtung der Rheinzollgerichte und des gerichtlichen Verfahrens in Rheinschiffahrtsangelegenheiten (RZGVO) vom 30. Juni 1834 in G S 1834, S. 136 ff u. in RHEINURKUNDEN I, Nr. 105, S. 306 - 313; vgl. CHRISTIAN ECKERT, S. 230; DiETER KISCHEL, Geschichte, S. 66 ff.

119 Das waren entweder der Friedensrichter in den Gebieten Rheinischen Rechts oder der Justizamtmann in den Gerichten zwischen Worms und Honnef oder aber bei den Land- und Stadtgerichten Duisburg, Wesel und Emmerich ein Land- bzw. Stadtgerichtsrat. Kritisiert als Vermischung der nationalen Gerichtsbarkeit mit der als international geplanten Rheinzollgerichtsbarkeit u. a. von GODEFROI, S. 494 ff (511); vgl DiETER KISCHEL, Geschichte, S. 70.

120 Nach $₫ 15$ II 17 ALR gehörten in Preußen die Fiskalprozesse nicht zur Polizeigerichtsbarkeit, sondern waren als besondere Verfahrensart im 35. Titel $\$ \$ 34 \mathrm{ff}$ der Preußischen Prozeßordnung = Teil I der Allgemeinen Gerichtsordnung (AGO) v. 1793, i. d. Fass. von 1818 geregelt; vgl. DIETER KISCHEL, Geschichte, S. 69. Durch Gesetz vom 24. April 1854 (G S 1854, S. 203 ff = RHEINURKUNDEN I, Nr. 271, S. 538f) wurde das Verfahren der Appellation geändert.

121 Vgl. CHRISTIAN ECKERT, S. $252 \mathrm{f}$.

122 Vgl. CHRISTIAN ECKERT, S. 253 ff; EBERHARD GOTHEIN, S. 246 ff.

123 Vgl. Christian ECKERT, S. 262. 
ländische Dampfschiffahrtsgesellschaften ${ }^{124}$ hervor, so wurde bereits 1825 eine preußischrheinische Dampfschiffahrtsgesellschaft ${ }^{125}$ gegründet, 1826 folgte in Mainz eine Dampfschiffahrtsgesellschaft vom Rhein und Main ${ }^{126}$ und 1827 eine Preußisch-Rheinische Dampfschiffahrtsgesellschaft ${ }^{127}$, die sich 1832 mit der Rhein-Main-Gesellschaft vereinig$\mathrm{te}^{128}$. Am 6. Mai 1841 spaltete sich in Köln die dort bestehende Preußische Rheindampfschiffahrtsgesellschaft, und die abtrünnigen Aktionäre taten sich in der „SchleppDampfschiffahrts-Aktiengesellschaft“ zusammen, die den Niederrhein bedienen sollte ${ }^{129}$. Um mit der niederländischen Dampfschiffahrtsgesellschaft in Ludwigshafen konkurrieren zu können, wandelte sich die Mannheimer Rangschiffahrtsgenossenschaft 1843 in eine Dampfschleppschiffahrtsgesellschaft um, die aus Schiffern und Kaufleuten bestand. Obwohl die Segelschiffer sich allmählich (seit etwa 1848) darauf einließen, statt von Pferden von Dampfern geschleppt zu werden, verdrängten die Dampfschleppzüge die alten Beurtfahrer und damit auch die Halfterer mit ihren Leinpferden ${ }^{130}$ auf Dauer vollständig ${ }^{131}$. In den Niederlanden wrackte man die großen Rheinsegelschiffe ab oder verkaufte $\operatorname{sie}^{132}$. Übrigens wurden durch Vertrag vom 2. September 1845 die Dampfschiffe in die Mainzer Akte einbezogen ${ }^{133}$, indem $\$ 1$ dieses Vertrages fortan nur ein Schiffsführerpatent und eine Konzession des Rheinuferstaates für den Betrieb der Aktiengesellschaft voraussetzte, um die Befugnis zur Dampfschiffahrt zu erfüllen ${ }^{134}$.

Daß das Comité der rheinischen Segelschiffer 1848 die politische Karte zu spielen versuchte und die Frankfurter Nationalversammlung aufforderte, für den Mittelstand „gegen die Geldaristokratie des 19. Jahrhunderts" Partei zu ergreifen "für die Arbeit gegen das Kapital" und "gegen die schiffahrttreibenden Handelsleute im Dienste des Mammons"135, den Gütertransport mit Hilfe von Dampfern zu verbieten, die Dampfschlepper zum Staatseigentum zu erklären und ihre Eigentümer von der Schiffahrt auszuschließen, war freilich vergeblich. Auch die nun folgenden Sabotageakte an Schleppdampfern, auf die gelegentlich sogar geschossen wurde ${ }^{136}$, waren folgenlose Verzweiflungsakte: Die rheinischen Dampfschleppschiffahrtsunternehmen machten geltend, daß sie nur in Reaktion auf die holländischen Unternehmen gehandelt hätten und daß die Mainzer, Mannheimer und Lud-

124 Vgl. Jürgen Heinz SCHAWACHT, S. $142 \mathrm{ff}$.

125 Vgl. Jürgen Heinz SCHAWACHT, S. $154 \mathrm{ff}$.

126 Vgl. den Bericht v. 15. Februar 1826 bei CHRISTIAN ECKERT, S. $410 \mathrm{ff}$ und die Vereinbarung v. 24. April 1826 ebenda S. 419f.

127 Vgl. den Fahrplan dieser Gesellschaft bei CHRISTIAn ECKERT, S. 437 ff; vgl. JÜrGEN HEINZ SCHAWACHT, S. $154 \mathrm{ff}$.

128 Vgl. den Vertrag bei CHRISTIAN ECKERT, S. 442 - 446.

129 Über weitere Dampfschiffahrtsgesellschaften vgl. JÜRGEN HEINZ SCHAWACHT, S. 156 ff.

130 Statistik bei CHRISTIAN ECKERT, S. 266.

131 Vgl. CHRISTIAN ECKERT, S. 256 ff; $263 \mathrm{ff}$.

132 Vgl. CHRISTIAN ECKERT, S. 259 ff; KARL OTTO SCHERNER, Rheinakten S. 594.

133 Vertrag zwischen den Rheinanliegerstaaten zur Einfügung des Supplementar-Artikels 18 und Aufhebung des bisherigen Artikels 52 der Mainzer Akte vom 2. Sept. 1845, ratifiziert 30. Mai 1846, bei GEORGES FRÉDÉRIC DE MARTENS, Nouveau Recueil Général Bd. IX, S. 172 und in RHEINURKUNDEN I, Nr. 219, S. $474-476$.

134 Wie Fn. 133, S. 474 f.

135 Zitiert bei CHRISTIAN ECKERT, S. 260.

136 Jahresbericht der Kommission 1848, S. 4, zitiert bei CHRISTIAN ECKERT, S. 261. 
wigshafener Aktionäre dadurch gezwungen waren sich anzuschließen. Mit einem Wort: Die Dampfschiffe setzten ihren Siegeszug fort.

\section{Die Konkurrenz der Eisenbahn}

Die Zugeständnisse, die Holland in der Mainzer Akte hinsichtlich der Schiffsabgaben gewährt hatte, erleichterten zwar die Rheinschiffahrt in den Niederlanden in gewissem Maße, doch war sie weiterhin beeinträchtigt. Deshalb vereinbarten die Niederlande und Preußen weitere Entlastungen im Vertrag vom 3. Juni $1837^{137}$, der am 21. Januar 1839 noch erweitert wurde ${ }^{138}$. Gleichwohl sannen Ludolf Camphausen und David Hansemann auf eine Umgehung der niederländischen Häfen und betrieben deshalb den Bau einer Bahn zum belgischen Hafen Antwerpen. Diese Strecke (der „Eiserne Rhein“) wurde 1841 eröffnet ${ }^{139}$. Vorher hatte schon Friedrich Wilhelm Harkort die Köln-Mindener Eisenbahn geplant, die 1847/48 als Bergisch-Märkische Eisenbahn ihren Betrieb aufnahm. Sie eröffnete den Waren aus Köln und den Erzeugnissen der Schwerindustrie des Ruhrgebietes, an deren Entwicklung Kölner Bankiers sich maßgeblich beteiligt hatten, den Weg zur Weser und nach Bremen.

Mit den Rangfahrten auf dem Rhein konkurrierten nicht nur die Dampfschlepper, sondern auch - neben der Bahn nach Antwerpen - weitere Eisenbahngesellschaften: Am 15. Februar 1844 wurde die linke Rheinstrecke zunächst bis Bonn eröffnet, am 21. Januar 1856 bis Rolandseck fortgeführt und im Jahre 1858 bis Koblenz verlängert ${ }^{140}$. Das veranlasste die Kölner und die Mainz-Düsseldorfer Dampfschiffahrtsgesellschaft, sich zu verständigen, was dann 1853 in einem Einigungsvertrag zwischen beiden Gesellschaften mündete ${ }^{141}$. Zu Beginn der 1860er Jahre lösten sich die letzten Rangfahrten auf, weil z. B. Eisenwaren, die bisher die Beurtfahrer von der Mosel und der Saar auf dem Rhein verfrachtet hatten, nun billiger mit der Eisenbahn von Koblenz aus weiterbefördert werden konnten $^{142}$.

\section{3. Änderungen der Mainzer Akte}

Die Mainzer Akte wurde in vielfacher Hinsicht durch die Entwicklung überholt: Im Laufe der Zeit wurden 20 Supplementar-Artikel zwischen den Vertragsstaaten vereinbart ${ }^{143}$.

137 Ratifiziert am 31. Juli 1837, Druck bei GEORGES FRÉDÉRIC DE MARTENS, Nouveau Recueil Bd. XIV, S. 250 und in RHEINURKUNDEN I, Nr. 131, S. 343 - 352.

138 Ratifiziert am 2. April 1839, Druck bei GEORGES FRÉDÉRIC DE MARTENS, Nouveau Recueil Bd. XVI, S. 410 und in RHEINURKUNDEN I, Nr. 152, S. 377.

139 Vgl. Jörg Engelbrecht, S. 252.

140 Die Strecke Rolandseck - Remagen wurde am 21. Jan. 1858, von Remagen bis Weißenthurm am 15. Aug. 1858 und Weißenthurm bis Koblenz Hbf am 15. Nov. 1858 eröffnet, vgl. Die Bundesbahn, Heft 3/1981, Beilage, S. 23f; KOCH/RÖHR, S. 30f. Dagegen wurde die rechte Rheinstrecke erst zwischen 1869 und 1874 ganz geschlossen (Die Bundesbahn, Heft 3/1981, Beilage, S. 62 ff).

141 Vgl. CHRISTIAN ECKERT, S. 276 ff.

142 Vgl. den Jahresbericht der Centralkommission für 1860, S. VI; CHRISTIAN ECKERT, S. 267. Für 1862 sagt der Bericht (1862, S. IX), daß die sog. Beurt- oder Rangfahrt nur dem Namen nach noch bestehe.

143 Vgl. RheinurKunden Bd. I, Nr. 111, S. 324, Vertrag v. 20. Nov. 1834 (Supplementar-Art. I IV, Oberlast, Schiffahrtsabgaben, Flöße); Nr. 132, S. 353, Vertrag v. 1. Aug. 1837 (Suppl.-Art. V IX, Schiffahrtsabgaben, Oberlast, Rheinschiffahrtsgerichte, Lade- und Löschverbot); Nr. 148, S. 
Man änderte die Vorschriften über die Schiffahrtsabgaben, die Oberlast, die Rheinschiffahrtsgerichte, die Schifferpatente und den Lotsenzwang. Die holländischen Zölle auf dem Strom fielen auf Grund des Vertrages vom 31. Dezember 1831 fort $^{144}$.

Da der deutsche Zollverein die Erleichterung von Handel und Wirtschaft in Deutschland betrieb, lag es nahe, den Rhein und die Schelde als wichtige Schiffahrtsstraßen einzubeziehen $^{145}$. Dem diente der Handels- und Schiffahrtsvertrag zwischen dem Zollverein und den Rheinanliegern vom 31. Dezember $1851^{146}$, der den Mitgliedern des Zollvereins Handelsfreiheit auf dem niederländischen Rhein brachte. Der Vertrag vom 12. Dezember 1860 setzte die letzten auf Privileg beruhenden Rheinzölle herab ${ }^{147}$. Der Handelsvertrag zwischen dem Zollverein und Frankreich vom 2. August $1862^{148}$ befreite die Schiffer der Parteien vom Zoll, und der Schiffahrtsvertrag vom gleichen Tage ${ }^{149}$ öffnete ihnen wechselseitig die Ströme Frankreichs und Deutschlands. Vor allem die Friedensverträge von 1866 haben schließlich die 1814 zugesicherte Freiheit des Rheins endgültig durchgeführt und die letzten Rheinzölle beseitigt ${ }^{150}$. Übrigens hatte schon die Additional-Konvention vom 3. April $1860^{151}$ den Sitz der Zentralkommission von Mainz nach Mannheim verlegt.

\section{Die Revidierte Rheinschiffahrtsakte von 1868}

368, Vertrag v. 17. Juli 1838 (Suppl.-Art. X, Schiffseichung); Nr. 160, S. 379, Vertrag v. 27. Juli 1839, Suppl.-Art. XI - XIII, Oberlast, entzündliche o. ätzende Stoffe, zulässige Einsenkung); Nr. 170, S. 405, Vertrag v. 21. Sept. 1840 (Suppl.-Art. XIV, XV, Zentralkommission, Oberlast); Nr. 210, S. 462, Vertrag v. 27. u. 30. Aug. 1844 (Suppl.-Art. XVI u. XVII, Schiffahrtsabgaben); Nr. 219, S. 474, Vertrag v. 2. Sept. 1845 (Suppl.-Art. XVIII, Befugnis z. Fahrt mit Dampfschiffen); Nr. 240, S. 495, Vertrag v. 9. Dez. 1848 (Suppl.-Art. XIX, Schifferpatente); Nr. 257, S. 521, Vertrag v. 8. Sept. 1851 (Suppl.-Art. XX, Lotsenzwang).

144 Vgl.. RiCHARD HENNIG, S. 86.

145 Und nicht nur das: „Der Handels- und Schiffahrts-Vertrag zwischen dem deutschen Zoll- und Handelsvereine einerseits und Belgien andererseits" vom 1. Sept. 1844 (G S 1844, S. 577 - 595) öffnete auch die Schelde dem internationalen Verkehr.

146 Verträge vom 31. Dez. 1851, ratifiziert am 7. Mai 1852, Druck: GEORGES FRÉDÉRIC DE MARTENS, Nouveau Recueil Général, Bd. XVI, 2, S. $216-237$ = G S 1852, S. $145-175=$ RHEINURKUNDEN I, Nr. 258, S. $521-533$.

147 Vgl. RiCHARD HENNIG, S. 86.

148 Handelsvertrag vom 2. Aug. 1862, ratifiziert 9. Mai 1865, Druck: GEORGES FRÉDÉRIC DE MARTENS, Nouveau Recueil Général, Bd. XIX, S. 286 - 294; Tarif S. 352 - 449 = RHEINURKUNDEN II, Nr. 313, S. 19f [nur Art. 9].

149 Schiffahrtsvertrag vom 2. Aug. 1862, ratifiziert 9. Mai 1865, Druck: GEORGES FRÉDÉRIC DE MARTENS, Nouveau Recueil Général, Bd. XIX, S. 286 - 294 = G S 1865, S. 450 - 463.

150 Die Friedensverträge zwischen Preußen und Baden v. 17. Aug. 1866, Druck: GEORGES FRÉDÉRIC DE MARTENS, Nouveau Recueil Général, Bd. XVIII, S. 333 = RHEINURKUNDEN II, Nr. 337, S. 71 [nur Art. 9]; mit Bayern v. 22. Aug. 1866, Druck: GEORGES FRÉDÉRIC DE MARTENS, Nouveau Recueil Général, Bd. XVIII, S. 336 = RHEINURKUNDEN II, Nr. 338, S. 71 [nur Art. 10] und Hessen v. 3. Dez. 1866, Druck: GEORGES FRÉDÉRIC DE MARTENS, Nouveau Recueil Général, Bd. XVIII, S. 352 = RHEINURKUNDEN II, Nr. 339, S. 71 [nur Art. 12].

151 Vertrag über l'article additionel, betr. den Sitz der Zentral-Kommission zwischen den Rheinuferstaaten vom 3. April 1860, ratifiziert 1. Mai/24. August 1860, Druck: G S 1860, S. $445-446=$ RHEINURKUNDEN, I, Nr. 298, S. 585. 
Die Fortschritte der Rheinfreiheit durch die Friedensverträge von 1866 mußten noch durch Verträge mit den Niederlanden und Frankreich ergänzt werden. Die verbliebenen Rheinuferstaaten Baden, Bayern, Frankreich, Hessen, Holland und Preußen ernannten deshalb eine Kommission, welche die Mainzer Akte überarbeiten und an die herrschenden Verhältnisse anpassen sollte. Man war sich inzwischen darüber einig geworden, daß der Staat sich in die freie Rheinschiffahrt so wenig wie möglich einmischen sollte. Deshalb kam die Revidierte Rheinschiffahrtsakte ${ }^{152}$ vom 17. Oktober 1868 (sog. Mannheimer Akte) mit 48 Artikeln (gegenüber 109 Art. der Mainzer Akte) aus. Die Änderungen gegenüber der Mainzer Akte von 1831 sind folgende:

Artikel 1 erklärte die völlige Freiheit der Schiffahrt auf dem Rhein zwischen Basel ,jusq'à la pleine mer" (bis in das offene Meer) für Schiffe aller Nationen (nicht nur für die Anrainer), wenn sie die Schiffahrtsakte und die Sicherheitspolizeivorschriften einhielten.

Artikel 3: Abgaben, die sich lediglich auf die „Beschiffung“ gründeten, sollten wegfallen.

Artikel 5: Alle Stapel- und Umschlagsrechte sollten nach wie vor aufgehoben bleiben.

Artikel $15 \mathrm{ff}$ : Die Erlangung der Rheinschifferpatente wurde neu geregelt und auf die Führung von Dampfschiffen erstreckt.

Artikel 22 ff: Die Schiffsuntersuchung und das Untersuchungszeugnis wurden beibehalten.

Artikel 27 ff: Sie enthalten Vorschriften über die Unterhaltung der Fahrrinne, der Einrichtungen für das Ein- und Ausladen, die Niederlage von Waren etc.

Artikel 33 ff: Die Gerichte hießen jetzt Rheinschiffahrtsgerichte. Art. 33 erlaubte nun das, was Preußen schon nach 1831 praktiziert hatte: die bestehenden Gerichte (meist Friedensgerichte) mit der Rechtsprechung in Rheinschiffahrtssachen zu beauftragen ${ }^{153}$.

Die Rheinschiffahrtsgerichte waren jetzt zuständig in Strafsachen für alle Zuwiderhandlungen gegen schiffahrts- und strompolizeilichen Vorschriften, in Zivilsachen für Gebührenfragen und - wie bisher bei Beschädigung von Dritteigentum, jedoch jetzt ohne Beschränkung auf fahrlässige unerlaubte Handlungen (Art. 34). Das beschleunigte Verfahren wurde in Art. 36 neu geregelt. Betrug der Streitwert mehr als 50 Franken, konnte wahlweise bei der Central-Kommission (Art. 43) oder beim Obergericht des Landes (Art. 38) Berufung eingelegt werden. Preußen erließ ein Ausführungsgesetz und beließ die Zuständigkeit für Berufungssachen beim dritten Senat des Appellationsgerichtshofes in Köln ${ }^{154}$.

Zusammen mit der revidierten Akte ${ }^{155}$ und dem Schlussprotokoll ${ }^{156}$ erließ die Zentralkommission am 3. Juni 1869 eine neue „Schiffahrts-Polizei-und Flossordnung für den

152 Revidierte Rheinschiffahrts-Akte zwischen Baden, Bayern, Frankreich, Hessen, Niederland und Preussen vom 17. Oktober 1868, ratifiziert am 17. April 1869, Druck: G S 1869, S. $798-837$ = GEORGES FRÉDÉRIC DE MARTENS, Nouveau Recueil Général, Bd. XX, S. $355-374=$ RHEINURKUNDEN II, Nr. 350, S. $80-106$.

153 Vgl. DiETER KISCHEL, Geschichte, S. 99.

154 Die Rheinschiffahrtsgerichte wurden für Preußen neu geregelt durch das „Gesetz betr. die Rheinschiffahrtsgerichte"v. 9. März 1870, Druck: G S 1870, S. 177 - 187 = RHEINURKUNDEN II; Nr. 366, S. $171-178 . \$ 5$ bestimmt den dritten Senat des Rheinischen Appellationsgerichtshofes zu Köln wie bisher - für alle preußischen Rheinschiffahrtsgerichte; vgl. DIETER KISCHEL, Obergericht, S. 346 ff; für das Verfahren der Rheinschiffahrtsgerichte: DIETER KISCHEL, Geschichte, S. $98 \mathrm{ff}$.

155 Auswechselung der Ratifikationsurkunden am 17. April 1869, bei GEORGES FRÉDÉRIC DE 
Rhein“157 und die „Verordnung über den Transport entzündlicher, ätzender und giftiger Stoffe auf dem Rheine“158.

Die privatrechtlichen Artikel der Mainzer Akte waren überflüssig geworden, Artikel 48, weil es keine Rangfahrten mehr gab und die Vertragsfreiheit allgemein galt; die Artt. 53, Abs. III, IV und 56 der Mainzer Akte mit zivilrechtlichem Inhalt waren überholt, weil ihre Rechtssätze jetzt in der Schiffahrtspolizeiordnung geregelt waren. Da sich die Staaten möglichst in die Freiheit der Rheinschiffahrt nicht einmischen wollten, sagte die preußische Denkschrift wohl mit Recht, privatrechtliche Vorschriften seien überflüssig, soweit sie mit denen der Rheinuferstaaten übereinstimmten, oder unzulässig, soweit sie ihnen widersprachen ${ }^{159}$. Privatrecht war nunmehr das kodifizierte Recht der Einzelstaaten.

Auf dem ganzen linken Rheinufer galt französisches Recht, nämlich der Code civil von 1804 und der Code de commerce von 1808: Sie galten im französischen Elsass, in der bayerischen Pfalz, in Baden als Badisches Landrecht und Handelsgesetz (das nur eine Übersetzung des Code civil bzw. Code de commerce war), in Rheinhessen und im linksrheinischen Preußen als Rheinisches Recht. Auch am bergischen Rheinufer (rechtsrheinisch von Honnef bis südlich Duisburg) galt ebenfalls französisches Recht. In den Niederlanden war das Wetboek van Koophandel $(1831,1838)$ stark französisch beeinflusst. Lediglich im ehemaligen Herzogtum Kleve (von Duisburg bis unterhalb Emmerichs) galt das preußische Allgemeine Landrecht (ALR) und von oberhalb Worms bis Honnef gemeines Recht $^{160}$. Den ganzen Rhein entlang herrschte also fast nur französisches Recht, und - was schwerer wog - es bildete die Rechtsgrundlage der Wirtschaft in den Handelszentren Straßburg, Mannheim, Mainz und Köln und in den niederländischen Häfen.

Das änderte sich erst, als 1861 das ADHGB in Kraft trat. In gewisser Weise war das ein Rückschritt, weil nun nicht mehr das fortschrittliche französische Recht, sondern das Landfrachtrecht galt, das nach dem ADHGB auch auf Binnenschiffe anzuwenden war und auf dem preußischen Entwurf vom 17. Jan. 1857 für ein Handelsgesetzbuch beruh$\mathrm{te}^{161}$. Was im ADHGB nicht geregelt war, richtete sich allerdings weiterhin nach französischem Recht ${ }^{162}$.

\section{Ausblick}

MARTENS, Nouveau Recueil Général XX, S. 355 und in RHEINURKUNDEN II, Nr. 350, S. 80 100.

156 Druck in RHEINURKUNDEN II, Nr. 350, S. 100 - 106.

157 Schiffahrts-Polizei-und Flossordnung v. 3. Juni 1869, Druck für Preußen im Amtsblatt Coblenz, Nr. 28 v. 6. August $1869=$ RHEINURKUNDEN II, Nr. 352, S. $118-150$. Zur Rechtsprechung des Rheinschiffahrtssenats des Kölner Oberlandesgerichts vgl. WILFRIED BEMM, S. 79 ff.

158 Verordnung der Zentral-Kommission für die Rheinschiffahrt betr. den Transport entzündlicher, ätzender und giftiger Stoffe auf dem Rheine v. 3. Juni 1869, Druck wie Fn. 157, RHEINURKUNDEN II, Nr. 353, S. $150-155$.

159 Preußische Denkschrift v. 17. Oktober 1868, in: RHEINURKUNDEN II, Nr. 351, S. 107, Vorspruch.

160 Vgl. FRIEDRICH BLUME, Rechtsquellen: zum rheinischen Recht, S. 131 ff; zum preußischen Recht, S. 137; J. B. EMIL v. Traut, S. 126; DieTER KISCHEL, Geschichte, S. 58; ROlF HinTZE, S. 2 ff; KARL OTTO SCHERNER, Rheinakten, S. $596 \mathrm{ff}$.

161 Über diese Einzelheiten der Beratungen zum ADHGB vgl. LuTZ, Protokolle, S. 515; RoLF HINTZE, S. $8 \mathrm{ff}$.

162 Vgl. KARL OTTO SCHERNER, Rheinakten, S. 598. 
Da der Rhein weiterhin die große Nord-Süd-Achse für den Warenverkehr blieb, war die Entwicklung 1868 nicht abgeschlossen. Preußen erließ am 9. März 1870 das Gesetz betreffend die Rheinschiffahrtsgerichte ${ }^{163}$, die das bisherige preußische Recht der Mannheimer Akte anpasste und fortschrieb. Weitaus einschneidender war die Gründung des Deutschen Reiches im Jahre 1871. Nach Art. 4, Ziff. 9 der neuen Reichsverfassung unterlag die Schiffahrt auf Wasserstraßen, die mehreren Staaten gemeinsam waren, der Aufsicht und der Gesetzgebung des Reiches. Allerdings machte das Reich von dieser Kompetenz keinen Gebrauch, so dass die Rheinuferstaaten nach wie vor die Verhältnisse Rheinschiffahrt regelten ${ }^{164}$. Nach 1871 wurde die Rechtsvereinheitlichung in Deutschland vorangetrieben. Am 11. April 1877 erging das Gerichtsverfassungsgesetz (GVG ${ }^{165}$, in Kraft seit dem 1. Okt. 1879), das auch die Rheinschiffahrtsgerichtsbarkeit änderte. Die Rheinschiffahrtsgerichte standen nämlich wegen der internationalen Rheinschiffahrtsakte außerhalb der nationalen Rechtsordnung, auch mußten die entsprechenden Gerichte der Bundesstaaten integriert werden. Das geschah, indem $\$ 14$, (damals $₫ 14$, Nr. 1) GVG den Rheinschiffahrtsgerichten eine besondere Stellung gewährte, sie nämlich als besondere Landesgerichte im Sinne von $\$ 4$ EGGVG bestehen ließ ${ }^{166}$. Damit hatten die Rheinuferstaaten freie Hand in der Gestaltung ihrer Rheinschiffahrtsgerichte. Da $\$ 12$ des Ausführungsgesetzes zum GVG die bisherigen Rheinschiffahrtsgerichte aufgehoben hatte und um diese Gerichte an die internationalen Verträge anzupassen, mußten die Rheinuferstaaten in der Folge neue Ausführungsgesetze zur Rheinschiffahrtsakte erlassen ${ }^{167}$. Dabei vertrauten sie die Rheinschiffahrtsgerichtsbarkeit ausgewählten Amtsgerichten an, die nach dem GVG seit 1879 die Friedensgerichte abgelöst hatten ${ }^{168}$.

Mit dem Gesetz über die Rheinschiffahrtsgerichte (GRSchG) vom 5. Sept. $1935^{169}$ machte das Reich von der ihm in Art. 7, Ziff. 19 der Weimarer Reichsverfassung verliehenen Kompetenz Gebrauch, die Rheinschiffahrtsgerichtsbarkeit reichseinheitlich zu regeln. Es verringerte die Zahl der Rheinschiffahrtsgerichte drastisch: Gab es vorher für den deutschen Teil des Rheins 48 Gerichte (davon 27 allein in Preußen), so blieben davon im neuen Gesetz nur noch sechs übrig: Kehl, Ludwigshafen, Mannheim, Mainz, St. Goar und Duisburg-Ruhrort. Von den beiden rheinischen Gerichten war Duisburg-Ruhrort wegen des großen Binnenhafens das führende Rheinschiffahrtsgericht und St. Goar bearbeitete die Unfälle, die sich in der gefährlichen Gebirgsstrecke des Mittelrheins häuften. Obergericht für die Schiffahrtsgerichte Konstanz, Kehl, Mannheim und Mainz wurde das

163 Druck: G S 1870, S. 177 ff und in Rheinurkunden II, Nr. 366, S. 171 - 178; vgl. DIETER KISCHEL, Geschichte, S. $114 \mathrm{ff}$.

164 Vgl. MaX MallincKrodt, S. 317f; Albrecht FuChS, S. 16f; DieTer KISCHEL, Geschichte, S. 125.

165 Gerichtsverfassungsgesetz für das Deutsche Reich vom 7. Februar 1877, RGB1 S. $41-76$.

166 Vgl. HaHN, Materialien zum GVG, I, 1 S. 49; vgl. DiETER KISCHEL, Geschichte, S. 126.

167 So z. B. Preußen durch das Gesetz betr. die Rheinschiffahrtsgerichte vom 8. März 1879 mit der Verordnung vom 1. Sept. 1879, betr. die Sitze und Bezirke dieser Gerichte, G S 1879 S. 129 ff und S. 609f; auch in Rheinurkunden II, Nr. 435, S. $245 f$ und Nr. 443, S. 249f; dort auch die Ausführungsgesetze der übrigen Bundesstaaten Nr. 432, 434 - 439, S. 234f, 245 - 250.

168 Vgl. STRAUCH, Rheinische Gerichte in zwei Jahrhunderten, 3. Kapitel.

169 Gesetz über die Rheinschiffahrtsgerichte (GRSchG) v. 5. Sept. 1935 (RGBl. I, S. 1142). 
Oberlandesgericht Karlsruhe, für die Schiffahrtsgerichte St. Goar und Duisburg-Ruhrort blieb es der dritte Zivilsenat des nunmehrigen Oberlandesgerichts Köln ${ }^{170}$.

Durch Note vom 14. November 1936 kündigte die nationalsozialistische Reichsregierung die internationalen Stromakten und fühlte sich seitdem an die Mannheimer Akte von 1868 und ihre späteren Änderungen nicht mehr gebunden ${ }^{171}$. Sie zog daraus die Folgerung, daß es keiner besonderer Gerichte in Rheinschiffahrtssachen mehr bedürfe und regelte deshalb das Verfahren in Binnenschiffahrtssachen reichseinheitlich: Das Gesetz vom 30. Jan. $1937^{172}$ schaffte in $\ 5$ deshalb den $₫ 14$, Nr. 1 GVG ab und ermächtigte in seinem $\ 4$ den Reichsminister der Justiz, einem Amtsgericht die Verhandlung und Entscheidung in Binnenschiffahrtssachen für den Bezirk mehrerer Amtsgerichte zuzuweisen. Davon machte dieser in der Verordnung vom 30. Januar 1937173 Gebrauch. In der Besatzungszeit nach 1945 hatte jede Zone ihre eigenen Vorschriften ${ }^{174}$.

Für die weitere Entwicklung gebe ich nur Stichworte:

> Das Binnenschiffahrtsverfahrensgesetz vom 27. September $1952^{175}$ führte wieder ein einheitliches Verfahren vor den Rheinschiffahrtsgerichten ein und löste die zuvor in den Besatzungszonen geltenden unterschiedlichen Verfahren ab;

$>$ das Abkommen über die Gliederung der Rheinschiffahrtsgerichte im Rheinstromgebiet der Bundesländer Baden-Württemberg, Hessen, Nordrhein-Westfalen und Rheinland Pfalz vom 21. Juli $1954^{176}$ stellte nahezu denselben Zustand her, wie er seit 1937 bestanden hatte.

> Kleine Revision der Mannheimer Akte vom 20. November 1963177,

$>$ Verfahrensordnung der Berufungskammer der Zentraldirektion vom 23. Oktober $1969^{178}$,

170 Zu dessen Rechtsprechung vgl. WILFRIED BEMM, S. 79 - 96.

171 Vgl. Begründung des Gesetzes über das Verfahren in Binnenschiffahrtssachen (= Amtliche Erlasse und Verordnungen), in: DJ 1937, S. 175f; vgl. bereits KOFFKA, in DJ 1936, S. 1801; DIETER KISCHEL, S. 175.

172 Gesetz über das Verfahren in Binnenschiffahrtssachen (GBSchS) v. 30. Jan.. 1937 (RGBl. I, S. 1417).

173 Verordnung zur Durchführung des Gesetzes über das Verfahren in Binnenschiffahrtssachen v. 30. Jan. 1937 (RGBl. I, S. 101f; vgl. DIETER KISCHEL, S. 177.

174 Dazu vgl. DiETER KISCHEL, Geschichte, S. 183 ff.

175 Gesetz über das gerichtliche Verfahren in Binnenschiffahrts- und Rheinschiffahrtssachen vom 27. Sept. 1952, BGBl I, S. 641, in Kraft seit 1. Oktober 1952; vgl. DIETER KISCHEL, Geschichte, S. 187 ff; DIETER KISCHEL, Obergericht, S. $348 \mathrm{f}$.

176 „Abkommen über die Gliederung der Rheinschiffahrtsgerichte im Rheinstromgebiet"vom 21. Juli 1954, in Kraft seit dem 1. Juli 1954, Druck: GV NW 1954, S. 263; vgl. DIETER KISCHEL, Geschichte, S. 197f; DIETER KISCHEL, Obergericht, S. 349.

177 „Übereinkommen zur Revision der am 17. Okt. 1868 in Mannheim unterzeichneten Revidierten Rheinschiffahrtsakte", vom 20. Nov. 1963, Druck: BGBI II, 1966, S. 561 - 568 und Gesetz dazu v. 6. Juli 1966, ebenda S. 560., vgl. DIETER KISCHEL, Geschichte, S. 202 ff; 251f; DIETER KISCHEL, Obergericht, S. 349.

178 Verfahrensordnung der Berufungskammer der Zentraldirektion vom 23. Oktober 1969, in Kraft seit 1. April 1970, Druck in BGBl II, 1970, S. 37; vgl. DIETER KISCHEL, Geschichte, S. $211 \mathrm{ff}$. 
Zusatzprotokoll zur revidierten Mannheimer Akte vom 25. Oktober 1972179, welches die Übertretungen nach dem neuen Gesetz über Ordnungswidrigkeiten vom 24. Mai $1968^{180}$ bestrafte.

Zusatzprotokoll Nr. 3 zur revidierten Mannheimer Akte vom 17. Oktober 1979, das die einheitliche Regelung von Geldbußen enthält ${ }^{181}$.

Wie nicht anders zu erwarten, ist auch das Rheinschiffahrtsrecht weiterhin in dauernder Bewegung, doch sind die Änderungen nach dem zweiten Weltkrieg bisher auf Randgebiete beschränkt. Die Entwicklung muß jedoch weiter beobachtet werden ${ }^{182}$.

\section{Quellen}

1. B GBl. I = Bundesgesetzblatt, Teil I (Jahr, Seite).

2. Blume, FRIEDRICH, Übersicht der in Deutschland geltenden Rechtsquellen, Bonn 1847.

3. BundesbahndireKTiOn KÖln (Hrsg.), Die Entwicklung des Streckennetzes der Kölner Eisenbahn-Direktionsbezirke bis zum 31. März 1981 (Beilage zum Aufsatz „Bundesbahndirektion Köln, gestern - morgen - heute“, in: Die Bundesbahn, Heft 3/1981.

4. Daniels, Heinrich Gottaried Wilhelm, Über das Stapelrecht zu Kölln und Mainz. Verfasst im Namen der Handlungskammer, von H. Daniels, Rechtsgelehrter und Professor bei der Centralschule in Kölln, Kölln 1804.

5. G S: = GeSETZESSA M M LU N G für die königlichen Preußischen Staaten, Berlin 1806 bis 1945.

6. HAHN, CARL, Die gesamten Materialien zu den Reichs-Justizgesetzen in acht Bänden, Band I: Materialien z. Gerichtsverfassungsgesetz, Abteilung I, II, 2. Aufl., hrsg. von EDUARD STEGEMANN, Berlin 1883, Neudruck Aalen 1983.

7. Herman, Heinrich, Sammlung der seit dem Reichs-Deputations-Hauptschluß vom 25. Februar 1803 in Bezug auf Rheinhandel und Schiffahrt erschienenen Gesetze, Verordnungen und allgemeinen Instruktionen, Mainz 1820; 1. Fortsetzung, Mainz 1828 [Gesetze].

8. Hofmann, Hanns Hubert, Quellen zum Verfassungsorganismus des Heiligen Römischen Reiches Deutscher Nation 1495 - 1815, Darmstadt 1976.

9. KLÜBER, Johann LUdWIG, Acten des Wiener Kongresses in den Jahre 1814 und 1815, Band II: Hefte 5-8, 2. Aufl. Erlangen 1817; Band III = Hefte 9 - 12, Erlangen 1815 [Akten].

10. KLÜBER, JOHANn LUDWig, Quellensammlung zu dem Oeffentlichen Recht des Teutschen Bundes, 3. sehr vermehrte Auflage, Erlangen 1830, Nachdruck Leipzig 1970 [Quellen].

179 Zusatzprotokoll zu revidierten Mannheimer Akte vom 25. Oktober 1972, Druck in BGBI II, 1974, S. 1385; BGBl I, 1975, S. 743; vgl. DIETER KISCHEL, Geschichte, S. 218 ff.

180 Ordnungswidrigkeitengesetz vom 24. Mai 1968 (BGBl I, S. 481), jetzt in der Fassung vom 1. April 1987 (BGBl I, S. 602), Druck (mit allen späteren Änderungen) auch in der Sammlung SCHÖNFELDER, Deutsche Gesetze, Nr. 94.

181 Zusatzprotokoll Nr. 3 zur revidierten Mannheimer Akte vom 17. Oktober 1979, in Kraft seit dem 1. September 1982, BGBl II 1980, S. 876; BGBl II 1982, S. 858; Änderung des Art. 32 der revidierten Mannheimer Akte; vgl. DIETER KISCHEL, Geschichte, S. 219, Fn. 623.

182 Zur neueren Entwicklung: DIETER KISCHEL, Obergericht, S. 350f; WILFRIED BEMM, S. 79 ff. 
11. LutZ, Johann Von (Hrsg.), Protokolle der Kommission zur Berathung eines allgemeinen deutschen Handelsgesetzbuches, Hamburg 1861.

12. DE MARTENS, GEORGES FRÉDÉRIC DE, Recueil des principaux traités d'alliance et d'autres actes remarquables etc. fondé par G. F. de M., Göttingen 1817 - 1835.

13. De Martens, Georges Frédéric De, Nouveau recueil des traités ... 13 Bde, Göttingen $1817-1842$.

14. V. MEYER, Philipp Anton Guido/Zoepfl, Heinrich (Bearb.), Corpus Iuris Confoederationis Germanicae oder Staatsacten für Geschichte und öffentliches Recht des Deutschen Bundes. Nach officiellen Quellen hrsg. von PH. A. G. V. PHILIPP ANTON GUIDO v. MEYER, ergänzt u. fortgeführt v. HeINRICH ZOEPFL, 1. u. 2. Theil: Staatsverträge: Frankfurt/M 3. Aufl. 1858, 1859; 3. Theil: Protokolle und Beschlüsse der Deutschen Bundesversammlung ebda 1865 [Staatsakten].

15. RGBl. = Reichsgesetzblatt (Jahr, Seite, seit 1921: RGBl. Teil I u. II)

16. RHEINURKUNDEN, Sammlung zwischenstaatlicher Vereinbarungen, landesrechtlicher Ausführungsverordnungen und sonstiger wichtiger Urkunden über die Rheinschiffahrt seit 1803, hrsg. v. d. Zentralkommission f. d. Rheinschiffahrt, Bd. I: 1803 - 1860; Bd. II: 1861 1918, München etc. 1918.

17. SAMMLUNG der Gesetze und sonstigen Vorschriften bezüglich der Rheinschiffahrt in den Rheinuferstaaten Baden, Bayern, Elsaß-Lothringen, Hessen, Niederlande und Preußen nach dem Stand am Schluß des Jahres 1868, hrsg. v. Rheinschiffahrts-Register-Verband, Frankfurt/M 1869.

18. ZEUMER, KARL, Quellensammlung zur Geschichte der deutschen Reichsverfassung, 2. Auflage, Tübingen 1913, Neudruck Aalen 1987.

\section{Literatur}

19. B ̈̈R, MAX, Die Behördenverfassung der Rheinprovinz, Bonn 1919.

20. Bärmann, JOHANneS, Die Freiheit der europäischen Binnenschiffahrt (Schriftenreihe der Deutschen Europa-Akademie 7), Mannheim 1950.

21. Baumgartner, Rudolf, Die Freiheit der Rheinschiffahrt. Ein Beitrag zur Rechtsgeschichte des internationalen Stromschiffahrtsrechtes, Bern 1926 (zugl. Diss. Bern 1924.

22. Bemm, Wilfried, Zur Rechtsprechung des Schiffahrtssenates, in: Rheinische Justiz. Geschichte und Gegenwart. 175 Jahre Oberlandesgericht Köln, hrsg. von DIETER LAUM/AdOlF KLEIN/DiETER STRAUCH, Köln 1994, S. 79 - 96.

23. Bouman, Pieter Jan, Der Untergang des holländischen Handels- und Schiffahrtsmonopols auf dem Niederrhein 1831 - 1851, in: VSWG Bd. 26 (1933), S. $244-266$.

24. BRUnNer, Hanns, Das Rheinschiffahrtsrecht nach Massgabe der Staatsverträge, Diss. iur. Würzburg 1919.

25. Chiesa, Pierre Albert, Le régime international du Rhin et la participation de la Suisse, Fribourg 1952.

26. Dollhoff, Josef, Die Kölner Rheinschiffahrt von der Römerzeit bis zur Gegenwart, Köln, 1980.

27. ECKERT, Christian, Die Rheinschiffahrt im 19. Jahrhundert, Leipzig 1900.

28. EICHHOFF, JOHANn JOSEPH, Topographisch-statistische Darstellung des Rheins mit vorzüglicher Rücksicht auf dessen Schiffahrt und Handlung ... Köln 1814 [Rhein].

29. Engelbrecht, JÖrg, Verkehrs- und Kommunikationsbeziehungen zwischen Köln und dem Niederrhein, in: Köln und die Niederrheinlande in ihren historischen Raumbeziehungen (15. - 20. Jahrhundert), hrsg. v. DIETER GEUENICH, Mönchengladbach 2000, S. 239 255. 
30. Ennen, Edith/HÖroldt, Dietrich Vom Römerkastell zur Bundeshauptstadt. Kleine Geschichte der Stadt Bonn, 4. Auflage, Bonn 1985.

31. Eysinga, Willem Jan Marie Van, Die Zentralkommission für die Rheinschiffahrt, Leiden 1936.

32. FrançOIS, S., Le rôle de la Commission Centrale pour la Navigation du Rhin dans le domaine règlementaire, in: 150 JAHRE ZENTRALKOMMISSION, S. 87 - 99.

33. Fuchs, AlbReCHT, Der Rheinschiffahrtsprozeß. Verfassung und Verfahren der deutschen Rheinschiffahrtsgerichte in Zivilsachen, Diss. iur. Heidelberg 1928.

34. Fuhrmann, Hans, Die völkerrechtlichen Grundlagen der Rheinschiffahrt, Diss. iur. Köln 1954.

35. Gaum, Friedrich Wilhelm, Bemerkungen über den Octroi-Vertrag und die Mainzer Schifferorganisation (nebst einer historischen Einleitung über das Schiffahrtswesen des Oberrheins), Heidelberg 1806.

36. Ghillany, Friedrich Wilhelm, Diplomatisches Handbuch. Sammlung der wichtigsten europäischen Friedensschlüsse, Congressacten und sonstigen Staatsurkunden vom Westphälischen Frieden bis auf die neueste Zeit, 2 Theile, Nördlingen 1853/55.

37. Godefroi, MiChael Hendrik, Les tribunaux pour la navigation du Rhin, in: Revue de droit international et de législation comparée, Bd. I (1869), S. $494 \mathrm{ff}$.

38. GönnenWein, OtTo, Das Stapel- und Niederlagsrecht, Weimar 1939 [Stapel].

39. GÖnnenWein, OTt o, Die Freiheit der Flußschiffahrt, Stuttgart etc. 1940 [Freiheit].

40. Gothein, EBERHARD, Geschichtliche Entwicklung der Rheinschiffahrt im 19. Jahrhundert, Leipzig 1903.

41. GrieWANK, KaRL, Der Wiener Kongress und die Neuordnung Europas 1814/15, Leipzig 1942.

42. Hennig, Richard, 1831 - 31. März - 1931 [100 Jahre Mainzer Akte], in: Zeitschrift für Binnenschiffahrt [ZfB], 1931, S. $84-86$.

43. HiNTZE, ROLF, Eine Darstellung der Entstehung des Binnenschiffahrtsgesetzes und der Entwicklung der Binnenschiffahrts-Bedingungen unter Berücksichtigung der Haftungsregelungen, Diss. iur. Würzburg 1965.

44. Hostie, Jan FRAnÇOIS Historischer Rückblick auf die Rechtslage auf dem Rhein, in: Der Rhein 1923, S. $171-174$.

45. HundertfünfZig Jahre Zentralkommission für die Rheinschiffahrt, hrsg. v. d. Zentralkommission f. d. Rheinschiffahrt durch KURT HILL, Straßburg, 1966.

46. Jollivet, Jean Baptiste-Moise, 1801: Du Thalweg du Rhin, considéré comme limite entre la France e l'Allemagne, an X, übersetzt; $A$. Crass „Betrachtungen über den Thalweg des Rheins in Hinsicht als Grenzscheide zwischen Frankreich und Deutschland; über die auf beiden Rheinufern angelegten Zölle, Mauthen und die den zwei Städten Mainz und Kölln zustehende Stapelgerechtigkeit“, Mayence (A. Crass), Vendémiaire an X (1801).

47. KisCHEL, DieTER, Die Geschichte der Rheinschiffahrtsgerichtsbarkeit von 1804 bis in die Gegenwart, unter besonderer Berücksichtigung der rheinpreußischen Verhältnisse (Steuer, Wirtschaft und Recht, Bd. 61), Berg. Gladbach etc. 1990 (zugleich Diss. iur. Köln 1989) [Geschichte].

48. Kischel, DieTer, 175 Jahre OLG Köln - 160 Jahre Rheinschiffahrtsobergericht. Zur Geschichte der Rheinschiffahrtsgerichtsbarkeit im Allgemeinen und des Rheinschiffahrtsobergerichtes Köln im Besonderen, in: Rheinische Justiz. Geschichte und Gegenwart. 175 Jahre Oberlandesgericht Köln, hrsg. von DieTER LAUM/ADOLF KLEIN/DiETER STRAUCH, Köln 1994, S. 335 - 351 [Obergericht). 
49. Johann Ludwig KLÜBER, Johann Ludwig, Das Staatsrecht des Rheinbundes, 1808 [Staatsrecht].

50. JOHANn LUDWIG KLÜBER, JOHANn LUDWIG, Öffentliches Recht des teutschen Bundes und der Bundesstaaten, 4. Aufl. Frankfurt/M 1840, Nachdruck Frankfurt/M 1970, [Öff. Recht].

51. KOCH, KARL-WILHelm/RÖHR, GuSTAV F., Der Rhein. Verkehrsweg im Herzen Europas. Schienenwege und Schiffahrt, Krefeld 1985.

52. KOFFKA, EMIL, Die Schiffahrtsgerichtsbarkeit nach dem 14. November 1936, in: Deutsche Justiz 1936, S. $1801 \mathrm{f}$.

53. KÖPPEN, URSUla VON, Die Geschichte der Zentralkommission und die Rechtsordnung der Rheinschiffahrt, in: Zeitschrift für Binnenschiffahrt [ZfB] 1966, S. 331 - 337; auch in HUNDERTFÜNFZIG JAHRE ZENTRALKOMMISSION, S. $1 \mathrm{ff}$.

54. Krause, Gerhard, Die internationalen Stromschiffahrtskommissionen, Berlin 1931.

55. KUSKE, BRUnO, Die Rheinschiffahrt zwischen Köln und Düsseldorf vom 17. - 19. Jahrhundert (Beiträge zur Geschichte des Niederrheins, Bd. 20), Düsseldorf 1906.

56. LeDeRle, AlFRED, Das Recht der internationalen Gewässer unter besonderer Berücksichtigung Europas, Mannheim etc. 1920.

57. LingelbaCH, GeRHARD, Art. Zollverein, in: Handwörterbuch zur deutschen Rechtsgeschichte [HRG], Bd. V (1996), Sp. 1769 - 1771.

58. LoOs-Corswarem, Clemens v. Zum Stapelrecht von Köln und der Schiffahrt auf dem Niederrhein in der frühen Neuzeit, in: EN GELBRECHT, J ÖRG 2000 (Nr. 29), S. 323 338.

59. Mallinckrodt, Max, Art. Rheinschiffahrt, in: Wörterbuch des deutschen Staats- und Verwaltungsrechts, Bd. III (O - Z), 2. Auflage, Tübingen 1914.

60. Moser, Johann Ja KO B, Neues Teutsches Staatsrecht, Bd. 16, Teil 9: Von der Landshoheit in Ansehung Erder und Wassers, und was damit einen Zusammenhang hat, Neudr. D. Ausgabe 1773, Osnabrück 1968.

61. Nau, Bernhard, SEBastian V., Beiträge zur Kenntnis und Beförderung des Handels und der Schiffahrt, Bde I - III, Mainz 1818 - 23, Bd. V, Mainz 1825[Beiträge].

62. Ockhart, Josef Franz, Geschichtliche Darstellung der frühern und spätern Gesetzgebung über Zölle und Handelsschiffahrt des Rheines, Stuttgart etc. 1842.

63. HeinRiCH BERnhard OpPEnheim, , Der freie deutsche Rhein. Geschichte und staatsrechtliche Entwicklung der Gesetzgebung des Rheines, Stuttgart etc. 1842.

64. Peters, MaX, Das Jahrhundert d. Rheinschiffahrtsakte, in: Der Rhein 1931, S. 59 - 61.

65. ROßMÜLLER, MARGOT, Die Gerichtsbarkeit für die Rheinschiffahrt, Diss. iur. Köln 1952.

66. SCHAWACHT, JÜRGEN HEInZ, Schiffahrt und Güterverkehr zwischen den Häfen des deutschen Niederrheins (insbesondere Köln) und Rotterdam vom Ende des 18. bis zur Mitte des 19. Jahrhunderts (1794 - 1850/51), Köln 1973 (zugl. Diss. rer. pol. Köln 1971).

67. SCHERNER, KARL OTto, Zur Rechtsgeschichte der Rheinschiffahrt bis zur Wiener Schlussakte, in: Probleme des Binnenschiffahrtsrechts IV, Duisburg 1985, S. 41 - 61; wieder in Nr. 70, S. 367 - 413 (dieses hier zitiert: [Rheinschiffahrt]).

68. SCHERNER, Karl OtTo, Die Begründung des modernen Rheinschiffahrtsrechts im 19. Jahrhundert, in: Probleme des Binnenschiffahrtsrechts, Bd. V, 1980, hrsg. v. Günther Wüst/Egon Lorenz/Karl Otto Scherner/Walter Müller, Heidelberg 1988, S. 65 - 98 [Begründung]. 
69. SCHERnER, Karl OTto, Die Rheinakten von 1831 und 1868. Die Freiheit der Rheinschiffahrt und das Verhältnis von Integration und Privatrecht um die Mitte des 19. Jahrhunderts, in: Zeitschrift für Europäisches Privatrecht, München 1997, S. 58 - 85; wieder in: Nr. 70, S. 579 - 606 (dieses hier zitiert: [Rheinakten]).

70. Scherner, Karl Otto, Handel, Wirtschaft und Recht in Europa (Gesammelte Aufsätze), Goldbach 1999.

71. Schirges, Georg, Der Rheinstrom. Ein Beitrag zur Kenntnis der Geschichte, Handelsstatistik und Gesetzgebung des Rheins, nebst Schiffahrtsakte von 1831, Mainz 1857.

72. SCHMitZ, JosEF, Die Entstehung der heutigen Rheinschiffahrtsgerichtsbarkeit und das Oberlandesgericht Köln als Rheinschiffahrtsobergericht, in: Recht und Rechtspflege in den Rheinlanden, hrsg. Josef Wolffram/Adolf Klein, Köln 1969, S. 301 - 306.

73. Schönfelder, Heinrich, Deutsche Gesetze. Sammlung des Zivil-, Straf- und Verfahrensrechts, Loseblattausgabe, München, Stand: Juli 2006.

74. SCHOLL, LARS U., Die Mainzer Rheinschiffahrtsakte vom 31. März 1831. „Übereinkunft unter den Uferstaaten des Rheines und auf die Schiffahrt dieses Flusses sich beziehende Ordnung“, in: Deutsche Schiffahrt. Informationen des Fördervereins Deutsches Schiffahrtsmuseum 1/1981, S. $21-24$.

75. SCHWAnn, MathieU, Geschichte der Kölner Handelskammer, Köln 1906.

76. Steins, HeinRiCh, Die Rheinschiffahrt von Köln bis Mainz vom 15. bis zum 19. Jahrhundert, Diss. phil. Bonn 1911.

77. Strauch, Dieter, Rheinische Gerichte in zwei Jahrhunderten. Die Entwicklung der ordentlichen Gerichtsbarkeit in der Rheinprovinz und ihren Nachfolgestaaten von 1798 2005, Düsseldorf 2007.

78. Traut, J. B. Emil v., die Zentralkommission für die Rheinschiffahrt und ihre Rechtsprechung von 1832 - 1911, 2. Aufl. Straßburg 1912.

79. VAlotTON, James, Du régime juridique des cours d'eau internationaux de l'Europe centrale, in: Revue de droit international et de législation comparée Bd. 15, 1913, S. 271 306.

80. Verdross, Alfred, Völkerrecht, 5. Auflage, Wien 1964.

81. VitÁnYi, BÉ LA, The International Regime of River Navigation, Alphen aan Rijn 1979.

82. VÖLDERndorfF, OTto FRHR. v., Die richterliche Tätigkeit der Centralkommission für die Rheinschiffahrt von 1832 - 1894, Frankfurt/M 1894.

83. Walther, H., Les tribunaux de la navigation du Rhin et l'activité jurisdictionelle de la Commission Centrale, in: HundertFünfZig JAHRE ZENTRALKOMMission (Nr. 45), S. $113-125$.

84. Heinz WASSERMEYER, Heinz, Heinrich Gottfried Wilhelm Daniels u. der Kölner Stapel, in: GEORG SCHIRGES (Nr. 71), S. 285 - 300.

85. WerkMÜller, Dieter, Art. Leinpfad, in: Handwörterbuch zur deutschen Rechtsgeschichte $\left[\mathrm{HRG}^{1}\right]$, Bd. II (1978), Sp. 1831 - 1837; bearb. v. KARL OTTO SCHERNER in: $\mathrm{HRG}^{2}$, Bd. III, 2016, Sp. $827-829$. 